\title{
Alterations to parvalbumin-expressing interneuron function and associated network oscillations in the hippocampal - medial prefrontal cortex circuit during natural sleep in APPNL-G-F mice
}

Erica S Brady ${ }^{1}$, Jessica Griffiths ${ }^{1,2}$, Lilya Andrianova ${ }^{1,3}$, Takashi Saito ${ }^{4}$, Takaomi C Saido ${ }^{5}$, Andrew D Randall ${ }^{1,6}$, Francesco Tamagnini ${ }^{1,2}$, Jonathan Witton ${ }^{1}$ and Michael T Craig ${ }^{1,3}$

1. Institute of Biomedical and Clinical Science, University of Exeter Medical School, Prince of Wales Road, Exeter, EX4 4PS, England, UK

2. School of Pharmacy, University of Reading, Whiteknights, Reading RG6 6LA, UK

3. School of Psychology and Neuroscience, College of Medical, Veterinary and Life Sciences, University of Glasgow, Glasgow G12 8QQ, Scotland, UK

4. Department of Neurocognitive Science, Institute of Brain Science, Nagoya City University Graduate School of Medical Sciences, Japan

5. Laboratory for Proteolytic Neuroscience, RIKEN Center for Brain Science, Saitama 351-0198, Japan

6. School of Physiology and Pharmacology, University of Bristol, Bristol BS8 1TD, UK

Correspondence to mick.craig@glasgow.ac.uk or j.witton@exeter.ac.uk 


\section{Abstract}

In the early stages of Alzheimer's disease (AD) the accumulation of the peptide amyloid- $\beta$ $(A \beta)$ damages synapses and disrupts neuronal activity, leading to the dysfunction of neuronal oscillations associated with cognitive function. This is thought to be largely due to impairments in CNS synaptic inhibition, particularly via parvalbumin (PV)-expressing interneurons that are pivotal contributors to several key oscillations. During non-rapid eye movement (NREM) sleep, communication between the hippocampus and medial prefrontal cortex (MPFC) facilitates the reorganisation of newly acquired hippocampal memories into the cortex for long-term consolidation, a process named systems consolidation. This occurs through the temporal coupling of the cortical slow wave oscillation (SWO), sleep spindles and hippocampal sharp-wave ripples (SWRs); oscillations generated, in part, through the action of PV-expressing interneurons. Disturbances to NREM sleep and impairments to these oscillations occur in preclinical $A D$ and in mouse models of amyloidopathy, yet in-depth characterisation of how this circuit is affected is presently lacking. Therefore, using 16 monthold homozygous APP ${ }^{\mathrm{NL}-\mathrm{G}-\mathrm{F}}$ mice, we recorded the electrical activity of this circuit during natural sleep. Despite widespread A $\beta$ pathology, deficits in the SWO were not detected at this age, yet an increase in the amplitude of mPFC spindles and decrease in the power of hippocampal SWRs was identified. The former was associated with a decrease in the density of mPFC PVexpressing interneurons and the latter, was accompanied by an increase in the synchronisation of PV-expressing interneuron activity, as measured using two-photon $\mathrm{Ca}^{2+}$ imaging. Furthermore, although changes were detected in the local oscillations and networks, the temporal communication between prefrontal and hippocampal circuits was intact, potentially suggesting the early stages of circuit breakdown in response to pathological $A \beta$. 


\section{Introduction}

Alzheimer's disease (AD) is a progressive neurodegenerative disorder and the most prevalent form of dementia worldwide ${ }^{1}$. In the preclinical stages of the disease, preceding overt signs of cognitive decline, the build-up and aggregation of the peptide amyloid- $\beta(A \beta)$ causes detriment to the synapse and neuronal activity, before accumulating further to form the phenotypic extracellular plaque pathology. In turn, these detriments lead to the disruption of the synchronous communication between large groups of neurons that generates the neuronal oscillations, associated with cognitive function ${ }^{2-7}$. These impairments to the electrical activity of the brain can be measured in humans as hyperactivity of the hippocampus and areas of the default mode network $(\mathrm{DMN})^{8,9}$ and lead to generation of epileptiform activity ${ }^{10}$ and a general "slowing" of the EEG ${ }^{11}$. Strong evidence suggests that impaired inhibitory neurotransmission underlies this breakdown in circuit function ${ }^{12}$. In particular, parvalbumin (PV)-expressing interneurons have been shown to exhibit both hypoand hyperactivity in mouse models of amyloidopathy ${ }^{13-15}$, with the former being linked to a reduction in the amplitude of gamma oscillations and the emergence of epileptic spikes.

Sleep disturbances, particularly disruptions to the quality and duration of non-rapid eye movement (NREM) sleep, are also a common feature during the early stages of $A D$, and are strongly linked to cognitive decline ${ }^{16,17}$. Importantly, the long-term consolidation of memory is thought to occur during NREM sleep, with particular research emphasis being placed on the activity of the hippocampal - medial prefrontal cortex (mPFC) circuit, given its prominent roles in episodic and spatial memory, as well as higher-order executive functions ${ }^{18,19}$. The generation and temporal coupling of three cardinal neuronal oscillations; the cortical slow wave oscillation (SWO), spindles, and hippocampal sharp-wave ripples (SWR), facilitates the reorganisation of newly encoded hippocampal memories into distributed cortical networks for long-term storage, in a process called systems consolidation ${ }^{20,21}$. Perturbation of the SWO and spindles can be found in humans with $A D^{22,23}$, with similar disruptions to the SWO occurring in mouse models of amyloidopathy ${ }^{24-26}$, alongside impairments to SWRs ${ }^{27}$. However, while each cardinal oscillation has relevance to memory within this circuit, it is their temporal coupling that is thought to drive systems consolidation ${ }^{20,21}$. The function of this circuit in a mouse model of amyloidopathy has been probed before ${ }^{28}$, yet an in-depth 
characterisation is currently lacking. Given the importance of this circuit in memory consolidation, the evidence for its disruption, and that memory impairments are the main symptom of $A D$, it is important to understand the effect pathological $A \beta$ has upon its function.

With this goal in mind, we analysed the neuronal oscillatory activity of the hippocampal-mPFC circuit during NREM sleep in sixteen-month-old APP ${ }^{N L-G-F}$ mice. This APP mouse model was chosen as it overcomes the limitations of earlier, over-expression models by replacing the endogenous mouse amyloid precursor protein $(A P P)$ gene with a human version that harbours the Swedish, Arctic and Iberian mutations associated with familial forms of $A D^{29,30}$. In the more widely-studied over-expression models, mutated hAPP is expressed under a variety of different promoters, alongside mouse $A P P$, with non-physiological expression levels of APP potentially leading to artefacts and confounding preclinical studies. Indeed, it is hypothesised that over-expression models are one reason for the current translational gap that exists between $A D$ therapies that show efficacy in mice, but fail in phase III clinical trials ${ }^{31}$. Conducting experiments in next-generation mouse models that are not confounded by nonphysiological over-expression of APP should provide researchers a greater understanding of how $A \beta$ affects brain physiology. Moreover, it is believed that these models better represent the preclinical stage of $A D$, where therapeutic interventions may be more effective by being delivered prior to the occurrence of irreversible neurodegeneration and cognitive decline. 


\section{Methods}

\section{Animals}

Male and female, APP ${ }^{\mathrm{NL}-G-F / N L-G-F}$ mice (APP) at sixteen-months-old (originally obtained from the MRC Harwell Institute, bred internally, and maintained on a C57BL/6J background) and wildtype (WT) littermate controls were used in this study ${ }^{29,30}$. All animals were housed on a 12-hour light-dark cycle at a temperature of $22 \pm 2{ }^{\circ} \mathrm{C}$ and humidity of $45 \pm 15 \%$ and had ad libitum access to food and water. For electrophysiological studies, $\mathrm{N}=6$ mice per genotype were implanted with electrodes but due to post-operative complications, $N=5$ mice per genotype were used for experiments. $\mathrm{N}=7 \mathrm{WT}$ and $\mathrm{N}=8$ APP were used for immunohistochemical analysis. For two-photon imaging, APP mice were crossbred with mice expressing Cre recombinase in parvalbumin-expressing interneurons (PV-cre mice; The Jackson Laboratory, \#008069) to generate APPHOM::PV-cre (PVAPP) and APPWT::PV-cre (PVWT) mice. $\mathrm{N}=3$ PVWT and $\mathrm{N}=5$ PVAPP mice were used for experiments. All procedures were carried out in accordance with the UK Animals (Scientific Procedures) Act 1986 and EU Directive 2010/63/EU and were reviewed by the University of Exeter Animal Welfare and Ethical Review Body.

\section{Surgical implantation of electrodes}

Surgical procedures were performed using aseptic techniques. APP and WT mice were anesthetised with isoflurane and placed in a stereotaxic frame (Stoelting, IL, USA). An incision was made in the scalp to expose the skull and microscrews (Antrin, CA, USA) were inserted into the frontal, parietal and interparietal plates bilaterally to anchor the implant to the skull. $1 \mathrm{~mm}$ diameter craniotomies were made over mPFC (in mm, from Bregma: $\mathrm{AP}=+1.75, \mathrm{ML}=$ +0.25 ) and dorsal CA1 (in $\mathrm{mm}$, from Bregma: $\mathrm{AP}=-2.0, \mathrm{ML}=+1.4$ ) and four-channel electrodes (Q1x4-5mm-200-177-CQ4, Neuronexus, MI, USA) were implanted to a depth (in $\mathrm{mm}$, from the brain surface) of 1.7 (mPFC) and 1.5 (CA1), respectively. Silver wire (World Precision Instruments, FL, USA) and silver conductive paint (RS Components, Corby, UK) were used to connect the ground channel of each electrode array to an anchor screw overlying the cerebellum. Light-curable dental cement (RelyX Unicem, Henry Schein, NY, USA) was used to secure the electrodes to the skull and anchor screws, and the scalp was closed around the 
base of the implant using a suture. Subcutaneous carprofen $(5 \mathrm{mg} / \mathrm{kg}$ ) was used for the management of post-operative pain as required.

\section{Electrophysiology data acquisition}

Recordings commenced 8 weeks after surgery. Animals were recorded in their home cage for three hours during the lights-on circadian epoch to enable recording of neural signals during sleep. An overhead light remained on throughout recording (440 lux). A Logitech colour webcam was fitted overhead $(30 \mathrm{~Hz}$ frame rate) and videos were recorded throughout neural signal acquisition.

Neural signals were acquired by connecting each electrode array to an Omnetics adapter (OM26, NeuroNexus) and headstage amplifier (RHD2000, Intan Technologies, CA, USA). The two headstages were connected to a dual headstage adapter (Intan) that allowed signals to be relayed along a single tether cable (Intan RHD SPI) to an OpenEphys acquisition board. Signals were bandpass filtered online at $0.1-7932 \mathrm{~Hz}$ and continuously recorded at $30 \mathrm{kHz}$. Signals recorded in CA1 in two mice (1 APP; 1 WT) were of poor quality (potentially due to poor grounding) and so were excluded from analysis.

\section{Verification of electrode locations}

At the end of experimental procedures, mice were deeply anaesthetised using an overdose of sodium pentobarbital $(300 \mathrm{mg} / \mathrm{kg}$ ) and electrolytic lesions were made at electrode sites. Mice were then transcardially perfused with $0.1 \mathrm{M}$ phosphate buffer (PB) followed by $4 \%$ paraformaldehyde (PFA) in 0.1 M PB. Brains were extracted, postfixed in 4\% PFA in $0.1 \mathrm{M} \mathrm{PB}$ overnight, and cryoprotected in $30 \% \mathrm{w} / \mathrm{w}$ sucrose in $0.01 \mathrm{M}$ phosphate buffered saline (PBS). Brains were cut into $40 \mu \mathrm{m}$ coronal sections using a freezing microtome (SM2010R, Leica Microsystems) and every fourth slice through mPFC and dorsal CA1 was mounted onto a slide and stained with DAPI. Sections were visualised using an epifluorescence microscope (Nikon Eclipse E800) and recording locations were confirmed from the lesions and electrode tracts. mPFC regions were defined based on previous literature ${ }^{32}$, with electrode positions found in both the anterior cingulate cortex (ACC) and prelimbic (PL) cortex (Supplementary Figure 1A, B). Hippocampal electrode placement was identified through histology and the changing 
polarity of SWRs in local field potential (LFP) traces recorded through the layers of CA ${ }^{33}$. Only electrodes located in Stratum pyramidale (Str.P) were used for analysis (Supplementary Figure $1 C, D)$.

\section{Viral injection surgery and two-photon imaging}

PVWT and PVAPP mice underwent similar surgical procedures as described above. The skull was exposed, and a craniotomy was made over dorsal CA1 (in mm, from Bregma: AP = -2.2, $M L,+2.0)$. Next, $250 \mathrm{nl}$ of AAV9/2-CAG-dlox-GCaMP6f-dlox-WPRE-SV40 (5x10 $12 \mathrm{GC} / \mathrm{ml})(\mathrm{VVF}$, University of Zurich) was infused into the CA1 region at a depth of $1.3 \mathrm{~mm}$ from the dura (rate: $50 \mathrm{nl} / \mathrm{min}$ ) using a pulled glass micropipette and microinjection pump (World Precision Instruments). Three weeks after viral injection, mice were implanted with an optical cannula overlying dorsal CA1, as previously reported ${ }^{34}$. Anaesthesia was induced using ketamine (73 $\mathrm{mg} / \mathrm{kg}) /$ medetomidine $(0.44 \mathrm{mg} / \mathrm{kg})$ and maintained using isoflurane. The skull was exposed, and a $3 \mathrm{~mm}$ diameter craniotomy was drilled, centred on the previous injection site. The dura was removed, and a column of cortex was aspirated whilst continuously irrigating with chilled cortex buffer (in mM: $\mathrm{NaCl} 125, \mathrm{KCl} 5$, glucose 10, HEPES 10, $\mathrm{CaCl}_{2} 2, \mathrm{MgSO}_{4} 2$ ). A stainlesssteel cannula ( $3 \mathrm{~mm}$ o.d.; $2.4 \mathrm{~mm}$ i.d.; $1.5 \mathrm{~mm}$ long) with a $3 \mathrm{~mm}$ diameter coverslip (Warner Instruments) affixed to one end (using NOA71 adhesive, Norland) was lowered into the craniotomy to the depth of the external capsule and was glued in position (Loctite). The skull was then sealed with dental cement (Simplex Rapid, Kemdent) and a metal head-fixation bar was affixed to the implant.

Recordings were performed under light (0.5-1\%) isoflurane anaesthesia 150-240 min after initial induction of anaesthesia for surgery. Mice were transferred to a two-photon microscope and the head-fixation bar was secured using a clamp. Body temperature was maintained at $37^{\circ} \mathrm{C}$ using a homeothermic blanket. A piezoelectric transducer beneath the thorax continuously monitored breathing rate as a proxy measure of anaesthetic depth, and the isoflurane dose was adjusted to maintain a consistent breathing rate between 150-200 breaths per minute. Images were acquired at 16x objective magnification (Nikon Plan Fluorite, 0.8 NA, $3 \mathrm{~mm}$ WD) using a microscope (Hyperscope, Scientifica) equipped with a Ti:sapphire pulsed laser (Chameleon Discovery, Coherent) and galvanometric scan mirrors. GCaMP6s was 
excited at $920 \mathrm{~nm}$ (power $<50 \mathrm{~mW}$ at the sample) and fluorescence was collected using a 500$550 \mathrm{~nm}$ emission filter. Images were acquired at $30 \mathrm{~Hz}$ using Scanlmage 4.0 software ${ }^{35}$. Spontaneous GCaMP6s signals were recorded continuously for 300 s, 200-300 $\mu \mathrm{m}$ beneath the external capsule (around the depth of CA1 Str.P). Several regions of interest (ROIs) (6.5 (3 7) [Med (Min Max)]) containing multiple GCaMP6s-positive neurons (5 (2 16) [Med (Min Max)]) were imaged per mouse.

\section{Electrophysiology data analysis}

All analysis was performed using custom-made scripts in MATLAB (Mathworks). Detailed analysis methods can be found in the supplementary material.

\section{Detection of slow wave sleep, the SWO, spindles and ripples}

The three stages of NREM sleep are typically grouped together in mice and named slow wave sleep (SWS $)^{36,37}$. To isolate SWS periods from non-SWS periods, signals were split into sections defined as either non-SWS (awake, microarousals, REM sleep) or SWS, using an algorithm based on previously published work ${ }^{38,39}$. LFP signals were split into 30 second epochs and a theta:SWO/delta (6-12 Hz:1-4 Hz) power ratio was created. If the power ratio in a 30 second epoch fell below a threshold (median +1 SD), and was also accompanied by a lack of movement, the epoch was considered SWS. If an epoch did not meet this requirement, it was considered to be non-SWS; only SWS epochs were used for subsequent analysis.

To analyse the dynamics of each of the three cardinal oscillations, custom-made detection scripts based on previously described methods were used to isolate and analyse individual oscillation cycles ${ }^{36,40-47}$. The SWO was detected by first low-pass-filtering the raw SWS signal (1.5 Hz) and all negative to positive zero-crossings were found. Two consecutive negativepositive zero-crossings within $0.25-2 \mathrm{~s}(0.5-1.25 \mathrm{~Hz})$ of each other and the largest peak and trough between them were used in the next stage of detection. Events were classed as a SWO if the peak was greater than the 60th percentile of all detected peaks and the total amplitude (peak-trough) was also greater than the 60th percentile of all detected amplitudes. 
For the detection of spindle $(9-16 \mathrm{~Hz})$ and ripple $(130-250 \mathrm{~Hz})$ events, the raw SWS signal was first bandpass filtered, z-scored and peaks were interpolated using cubic spline interpolation. Peaks that crossed a 3.5 SD threshold were identified and start and stop times of 1.5 SD either side were taken for the detection of spindles. A 6.5 SD threshold with 2 SD start and stop times were used for the detection of ripples. Events were then classed as spindles if they were between 0.5 and $2 \mathrm{~s}$ long and had an intra-spindle frequency of $11-15 \mathrm{~Hz}$ and as ripples if their duration was between $30-250 \mathrm{~ms}$ and had an intra-ripple frequency of $130-250 \mathrm{~Hz}$.

\section{Oscillation coupling}

Analysis windows spanning $2 \mathrm{~s}$ on each side of detected Down states were used to analyse the phase-amplitude coupling (PAC) ${ }^{48}$ between detected SWO events and gamma oscillations. Spindles were considered coupled to the SWO If the peak of a detected spindle event occurred within $1 \mathrm{~s}$ after the peak of a detected Down state. Sections of raw signal that began $2 \mathrm{~s}$ before the onset of the Down state and ended $2 \mathrm{~s}$ after the peak of the spindle event were used to analyse their PAC. Similar analysis methods were applied for the SWO and ripples, except a $0.5 \mathrm{~s}$ period after the ripple peak was used for PAC. If a ripple began within $2 \mathrm{~s}$ following the onset of a spindle, they were considered coupled.

\section{Two-photon imaging analysis}

Custom routines in MATLAB and FIJI software (National Institutes of Health, USA) ${ }^{49}$ were used. Mechanical drift was corrected by cross-correlation-based subpixel registration ${ }^{50}$ to the average of the first second (30 frames) of each image time series. Summed projection images were then used to manually draw ROls around visually identified cell bodies, and cellular fluorescence time series, $\mathrm{F}_{\mathrm{ROI}}(\mathrm{t})$, were constructed by averaging the ROI pixels in each frame. A correction for neuropil contamination was applied, following $F_{R O I}(t)=F_{R O I}$ measured $(t)-$

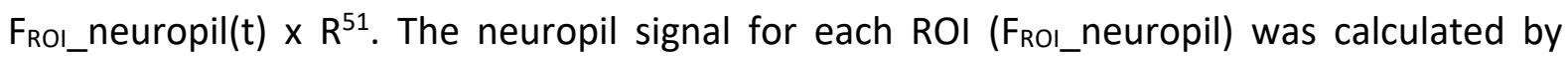
averaging the pixels within a $2 \mu \mathrm{m}$ annulus expanded around each ROI, excluding pixels within other cellular ROIs. R was the linear regression coefficient between the measured ROI signal and its neuropil signal. Slow drifts in the fluorescence time series were corrected by subtracting the 8 th percentile in a 30 second window centred on each time point, and relative fluorescence time series $(\Delta \mathrm{F} / \mathrm{F})$ were calculated as $\left(\mathrm{F}_{\mathrm{ROI}}(\mathrm{t})-\mathrm{F} 0\right) / \mathrm{F} 0$, where $\mathrm{F} 0$ was the median 
of $F_{R O I}(t)$ after drift correction. To detect fluorescence transients, we calculated the SD of the 20-80th percentile of $\Delta F / F$, and identified contiguous samples that exceeded $F 0+2 S D$, had a peak amplitude of at least F0 + 7SD. For correlation analysis, we calculated, for each cell, the mean pairwise zero-lag cross correlation between the cell's $\Delta \mathrm{F} / \mathrm{F}$ trace and the $\Delta \mathrm{F} / \mathrm{F}$ traces of all other simultaneously recorded cells.

\section{Immunohistochemistry}

Animals were transcardially perfused and brains were removed and sectioned, as described above. Sections were first washed in PBS with $0.3 \%$ Triton X-100 (PBS-T) before being immersed in 100 mM glycine in PBS-T. Sections were then washed in PBS-T and incubated in blocking solution ( $3 \%$ goat serum in PBS-T) for 1 hour. Primary antibodies (rabbit anti-PV,

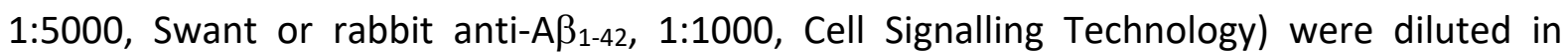
blocking solution, applied to the sections, and incubated overnight at $4^{\circ} \mathrm{C}$. The next day, sections were washed in PBS-T and incubated in blocking solution containing biotinylated goat anti-rabbit secondary antibody (1:250, Vector Laboratories) for 1 hour at room temperature. Sections were then rinsed in PBS-T and incubated in blocking solution containing DyLight 488 Streptavidin (1:250, Vector Laboratories) for 1 hour. Finally, sections were washed, mounted onto glass slides, and covered with glass cover slips with mounting medium containing DAPI.

Images were acquired at 10x magnification on a Leica confocal laser scanning microscope. Only the right hemisphere was imaged and each brain region was sampled at a similar distance from bregma between animals. Brain regions were defined according to published criteria $^{32}$ and the Allen Brain Atlas (https://mouse.brainmap.org/). To quantify the number of PV-expressing interneurons, the cell counter plugin was employed using FIJ software ${ }^{49}$. All cell counts were normalised to the brain region area. Widespread $A \beta_{1-42}$ pathology was observed in both the mPFC and hippocampus in APP mice (Supplementary Figure 2).

\section{Statistical analysis}

For two-photon imaging analysis, cell was used as the experimental unit ( $\mathrm{N}$ number) as amyloidopathy is a progressive pathology that affects different cells at different times, often 
dependent on proximity to $A \beta$ aggregates ${ }^{3}$. Nonetheless, cells within individual animals are not fully independent and may covary ${ }^{52}$. To test the effect of animal on the data, generalised linear mixed effects models (SPSS) were used that included 'genotype' as a fixed factor and 'animal' as a random factor. Values were modelled with a gamma distribution and log link function to account for skew in the distributions.

All other statistical testing was carried out using Prism 9 (GraphPad). Normality of the data was tested using a D'Agostino and Pearson test. A Mann-Whitney U-test of comparable ranks was used for nonparametric data. All non-parametric results are displayed as box plots showing the median, range and the inter-quartile range (IQR).

\section{Data availability}

All raw data that supports the findings herein are available from the corresponding author upon reasonable request. 


\section{Results}

\section{The power of the SWO and delta oscillation were unaltered in APP mice}

Across the cortex during SWS, the SWO $(0.5-1.25 \mathrm{~Hz})$ and delta oscillations $(1.25-4 \mathrm{~Hz})$ dominate ${ }^{53}$. Relative to WT mice, APP animals displayed no significant difference in the power of either the MPFC SWO or delta oscillation (Figure 1). The SWO is often misclassified as being a continuation of the delta oscillation ${ }^{54,55}$, when in fact the two are generated independently and potentially have different physiological roles ${ }^{53,56}$ : the SWO provides a temporal framework for spindles and SWR to nest within and is the temporal pacemaker of systems consolidation ${ }^{21,57}$, whereas there is evidence for delta oscillations playing a role in forgetting ${ }^{56}$. Therefore, further analysis was restricted to the SWO.

A

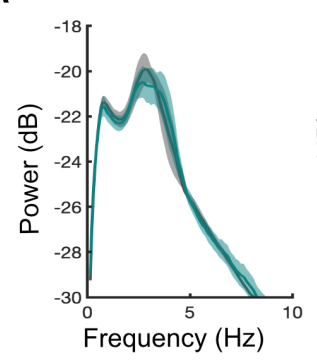

B

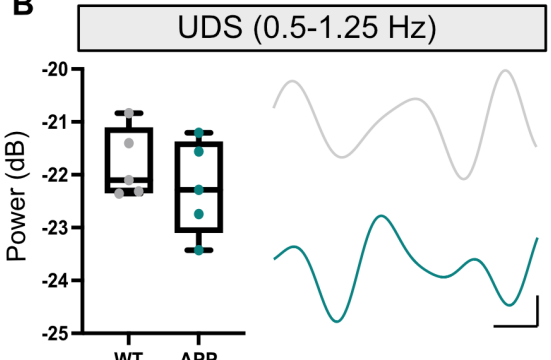

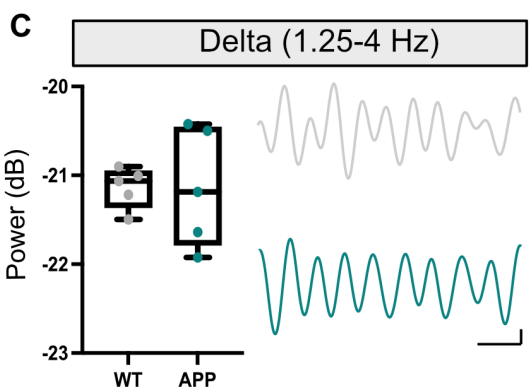

Figure 1 | The power of both UDS and delta oscillations in the MPFC is not altered in APP animals. A Average power spectra \pm SEM of both WT (grey) and APP (turquoise) mPFC LFPs during SWS, showing clear peaks in the UDS and delta frequency bands. B No statistically significant difference in the power of UDS was found in APP animals compared with WT (WT: -22.10 (-22.33- -21.12) vs APP: $-22.29(-23.09--21.38) \mathrm{dB}, \mathrm{U}=9, \mathrm{p}=0.54$, MannWhitney U test). Example traces show UDS bandpass filtered signals for both WT and APP mice. C No statistically significant difference in the power of delta oscillations was found in APP animals compared with WT (WT: -21.06 (-21.36- -20.95) vs APP: -1.19 (-21.78- -20.46) $d B, U=12, p>0.99$, Mann-Whitney $U$ test). Example traces show delta bandpass filtered signals for both WT and APP mice. Box plots show median, IQR and ranges. Descriptive statistics display median and IQR. Scale bars: $500 \mathrm{~ms}, 0.1 \mathrm{mV}$. 


\section{SWO-nested gamma oscillations were unaffected}

The SWO is composed of two distinct states, Up states and Down states (UDS), that reflect the rapid firing rate and subsequent hyperpolarisation of neurons, respectively ${ }^{58}$. Gamma oscillations $\left(30-120 \mathrm{~Hz}\right.$ ) can be found nested within SWO Up states ${ }^{59,60}$ (Figure $\left.2 \boldsymbol{A}, \boldsymbol{D}, \boldsymbol{E}\right)$. Given that humans with $A D$ exhibit impairments in the SWO and gamma oscillations ${ }^{61,62}$, which is recapitulated in many mouse models of amyloidopathy $13,14,24,25,63,64$ and is accompanied by dysfunction of the inhibitory interneurons ${ }^{13-15}$ that drive these oscillations, we hypothesised that both SWO and gamma oscillations would be disrupted in APP mice. However, no change to the duration and amplitude of Up states, Down states and combined UDS was identified in APP mice compared with WT controls, nor was there a change in the number of UDS events (Supplementary Figure 3). Furthermore, the power of both slow $(30-60 \mathrm{~Hz})$ and fast $(30-120$ $\mathrm{Hz}$ ) Up state nested-gamma oscillations was not altered in APP animals (Figure $\mathbf{2 B}, \mathbf{C}$ ). There was additionally no change in the phase-amplitude coupling (PAC) of both slow and fast gamma oscillations with the SWO (Figure 2D, E). 
A

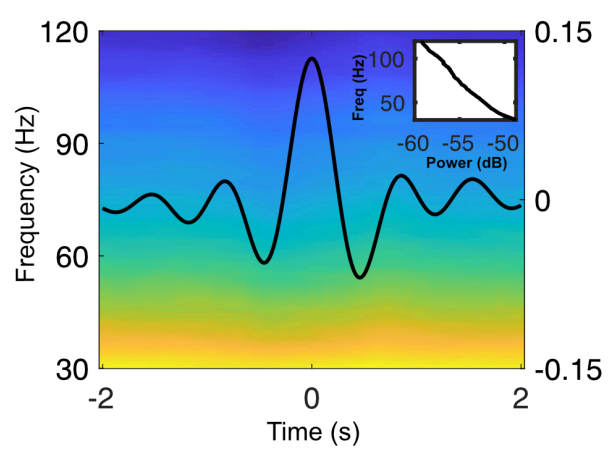

B

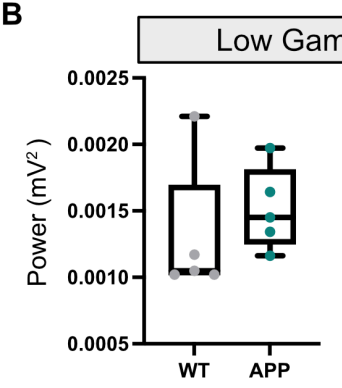

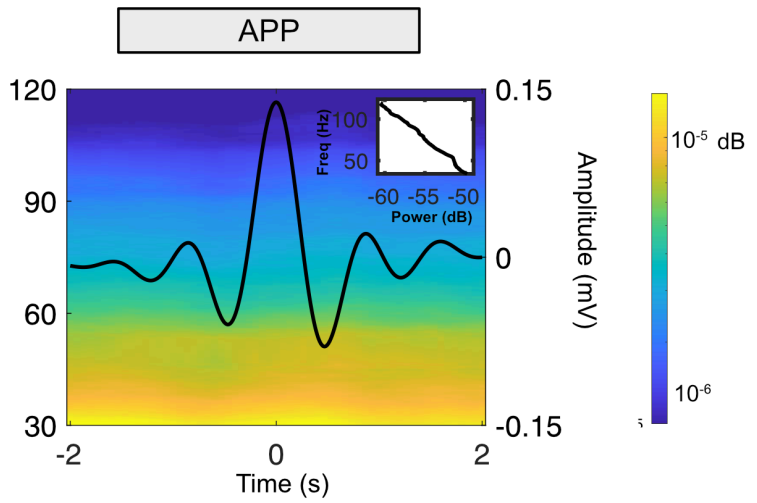

C

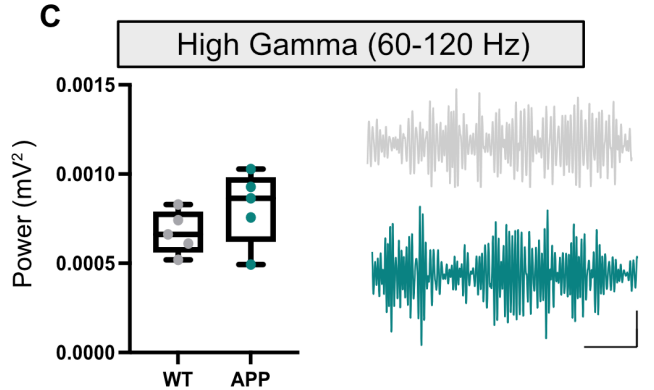

D
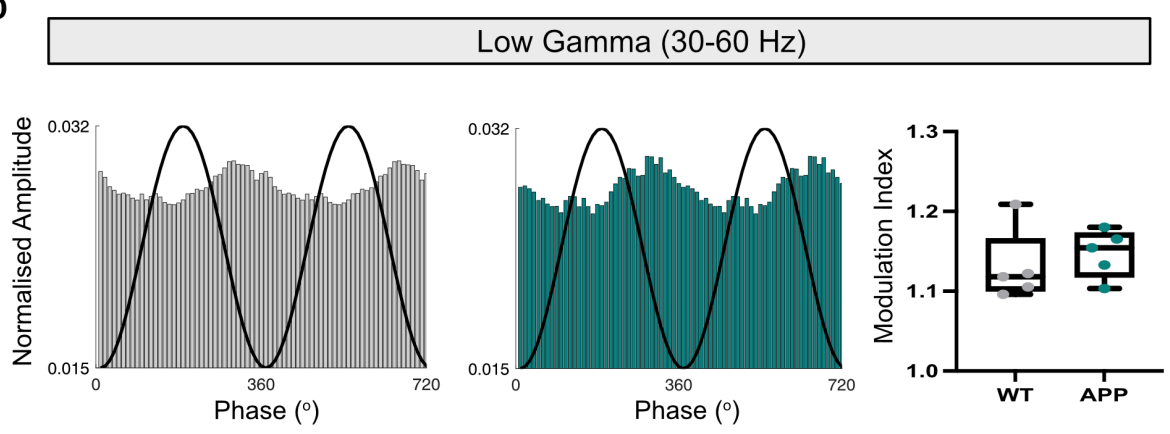

E

High Gamma $(60-120 \mathrm{~Hz})$
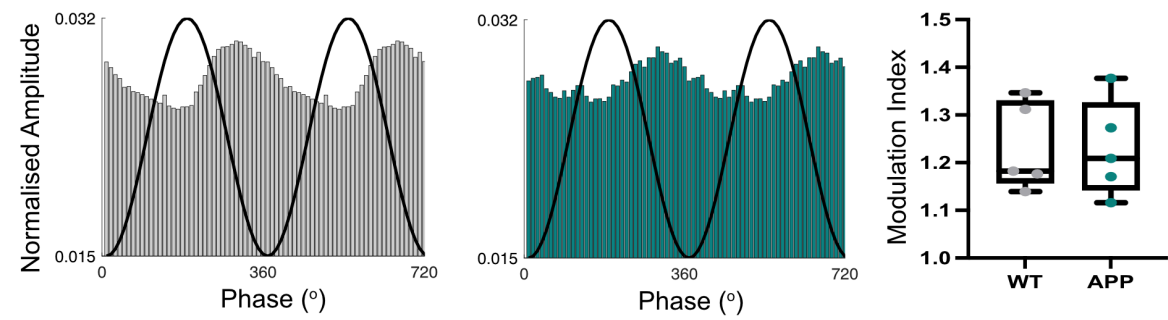

Figure 2 | UDS-locked gamma power and PAC is not altered in APP animals. A Example average UDS traces (black lines) overlaid on the corresponding LFP power spectrum showing the power of UDS-locked gamma oscillations for both WT and APP mice. Slight modulation of low gamma power can be visualised over the UDS time course. Indented plots (top right corner) display the mean gamma power over the time-course of the LFP power spectrum. B No statistically significant difference in the total power of UDS-locked low gamma oscillations was found in APP animals relative to WT (WT: 0.0010 (0.0010 - 
$0.0017)$ vs APP: $0.0014(0.0013-0.0018) \mathrm{mV}^{2}, \mathrm{U}=6, \mathrm{p}=0.2$, Mann-Whitney $\mathrm{U}$ test). Example traces show low gamma bandpass filtered signals for both WT (grey) and APP (turquoise) mice. C No statistically significant difference in the total power of UDS-locked high gamma oscillations was found in APP animals relative to WT (WT: 0.00066 (0.00057 0.00079 ) vs APP: $0.00087(0.00063-0.00098) \mathrm{mV}^{2}, U=6, p=0.22$, Mann-Whitney $U$ test). Example traces show high gamma bandpass filtered signals for both WT and APP mice. Scale bars: $200 \mathrm{~ms}, 40 \mu \mathrm{V}$. D No statistically significant difference in the PAC of UDS with low gamma oscillations was found in APP animals relative to WT (WT: $1.12(1.10-1.17)$ vs APP: $1.15(1.12-1.17) \mathrm{MI}, \mathrm{U}=8, \mathrm{p}=0.42$, Mann-Whitney $\mathrm{U}$ test). Shown are example histograms of the change in normalised low gamma amplitude (bars) over the phase of the UDS (black line) for both WT (left) and APP (right) mice. E No statistically significant difference in the PAC of UDS with high gamma oscillations was found in APP animals relative to WT (WT: $1.18(1.16-1.33)$ vs APP: $1.21(1.14-1.33) \mathrm{MI}, \mathrm{U}=12, \mathrm{p}=0.99$, Mann-Whitney $\mathrm{U}$ test). Shown are example histograms of the change in normalised high gamma amplitude (bars) over the phase of the UDS (black line) for both WT (left) and APP mice (right). Box plots show median, IQR and ranges. Descriptive statistics display median and IQR.

\section{An increase in spindle amplitude in the MPFC}

Spindles oscillations (11-15 Hz) are generated through recurrent thalamocortical (TC) projections ${ }^{65}$ (Figure $3 \boldsymbol{A}$ ). The number of spindle events linearly correlates with memory performance in humans ${ }^{40}$, with a decrease in the number of detected spindles found in humans with $A D^{23}$. A statistically significant increase in the amplitude and corresponding power of spindle events was found in the MPFC of APP animals relative to WT (Figure 3B, Cii), with no change to their duration, frequency, or occurrence (Figure $\mathbf{3 C i}$, iii, iv). PV-expressing interneurons (PV+ cells) in the thalamic reticular nucleus (TRN) are involved in the generation of spindles ${ }^{66}$, while in the mPFC these interneurons are important in the feed-forward inhibition of incoming TC inputs ${ }^{67}$. Therefore, the density of these interneurons in the TRN and mPFC was assessed using immunohistochemistry. No change in the PV+ cell density was found in the TRN of APP animals (Supplementary Figure $4 A, B$ ). However, a significant decrease in density was detected in the ACC sub-region of the MPFC (Supplementary Figure 
4C, D). This decrease in immunoreactivity could potentially affect the inhibitory capabilities of PV+ cells to gate the incoming TC inputs, producing the increase in spindle amplitude.

A
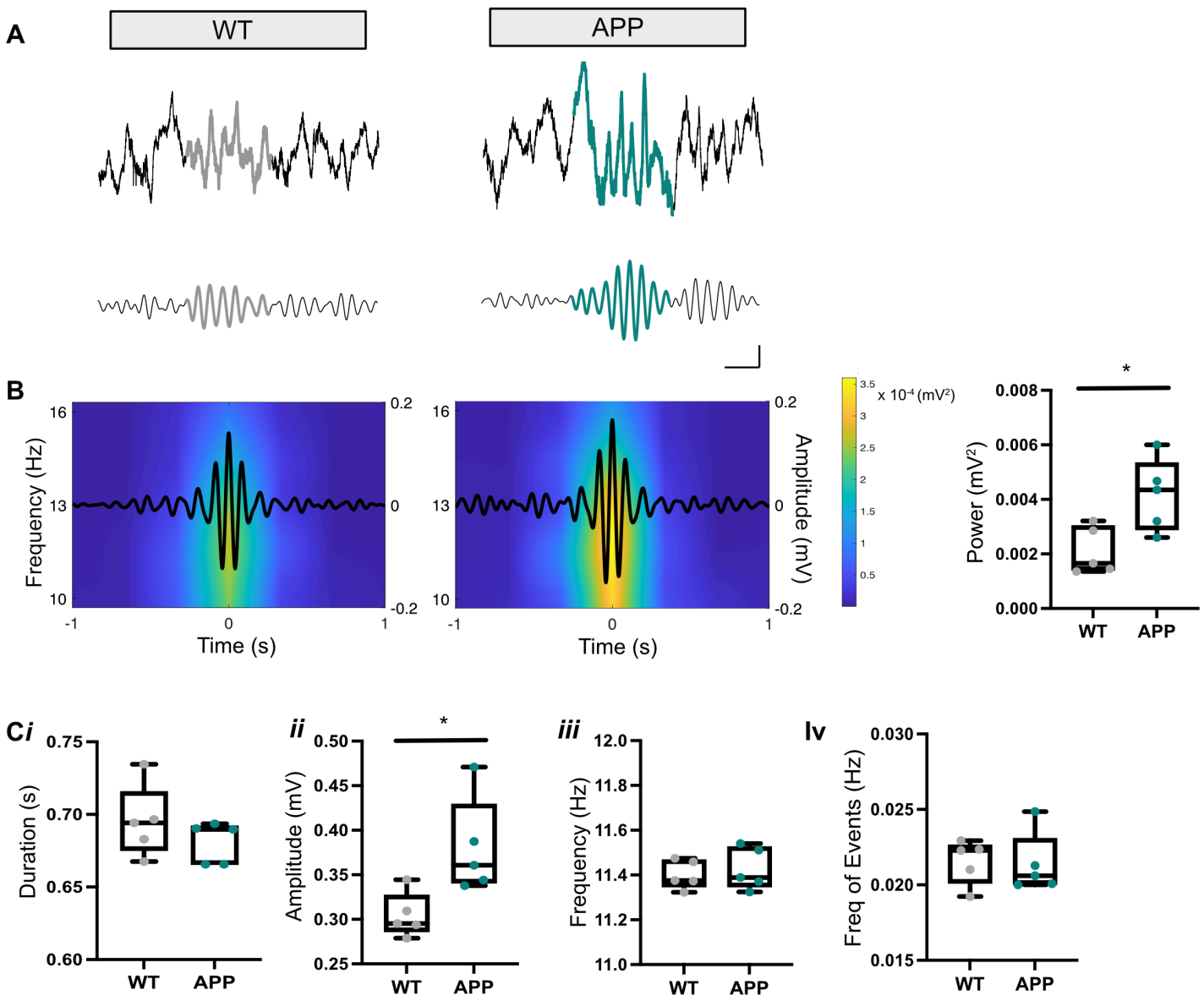

Figure 3 | The amplitude and power of mPFC spindles is increased in APP mice. A Example raw (top) and $11-15 \mathrm{~Hz}$ bandpass filtered (bottom) traces of detected spindle events (coloured segments) from WT and APP mice. Scale bars: 250 ms, $200 \mu \mathrm{V}$. B Example average spindle traces (black lines) overlaid on the corresponding LFP power spectrum showing average spindle power. A statistically significant increase in the average spindle power was found in APP mice compared with WT controls (WT: $0.0017(0.0014-0.0030)$ vs APP: $0.0043(0.0029-0.0053) \mathrm{mV}^{2}, \mathrm{U}=2.5, \mathrm{p}=0.03$, Mann-Whitney $\mathrm{U}$ test). C No statistically significant difference was found in APP animals relative to WT for the average spindle duration (WT: $0.69(0.68-0.0 .72)$ vs APP: $0.69(0.67-0.69) \mathrm{s}, U=6, p=0.22$, MannWhitney $U$ test) (i) or frequency (WT: 11.38 (11.35 - 11.47) vs APP: 11.39 (11.35 - 11.53) $\mathrm{Hz}, \mathrm{U}=10, \mathrm{p}=0.69$, Mann-Whitney $\mathrm{U}$ test) (iii) but a statistically significant increase in the average spindle amplitude was found in APP mice compared with WT controls (WT: 0.30 
$(0.29-0.33)$ vs APP: $0.36(0.34-0.43) m V, U=2, p=0.03$, Mann-Whitney $U$ test) (ii). No statistically significant difference was found in APP animals relative to WT for the incidence of detected spindle events (WT: $0.022(0.020-0.023)$ vs APP: $0.021(0.020-0.023) \mathrm{Hz}, \mathrm{U}=$ 10, $p=0.69$, Mann-Whitney $U$ test) (iv). Box plots show median, IQR and ranges. Descriptive statistics display median and IQR. ${ }^{*} p<0.05$.

\section{A decrease in ripple power and increased coordination of PV-expressing interneuron}

\section{activity in the hippocampus}

Hippocampal ripples (Figure $\mathbf{4 A}$ ) are associated with systems consolidation $68,69,70$, with disruptions to these oscillations impairing the consolidation of hippocampus-dependent memories $^{71}$. As SWRs are impaired in over-expression mouse models of amyloidopathy ${ }^{27}$, we hypothesised that disruptions would also be seen in APP mice. No statistically significant change in the duration and frequency of detected ripple events was identified in APP animals compared with WT (Figure 4Ci, iii). Interestingly, no change in the amplitude of ripples was detected either (Figure 4Cii), yet a significant decrease in the power was found to occur (Figure 4B). Furthermore, a trend towards an increase in the occurrence of detected ripple events was found in APP animals (Figure 4Civ). 
A
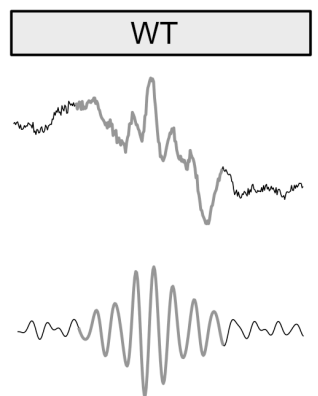
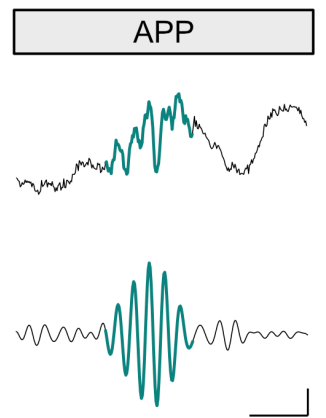

B
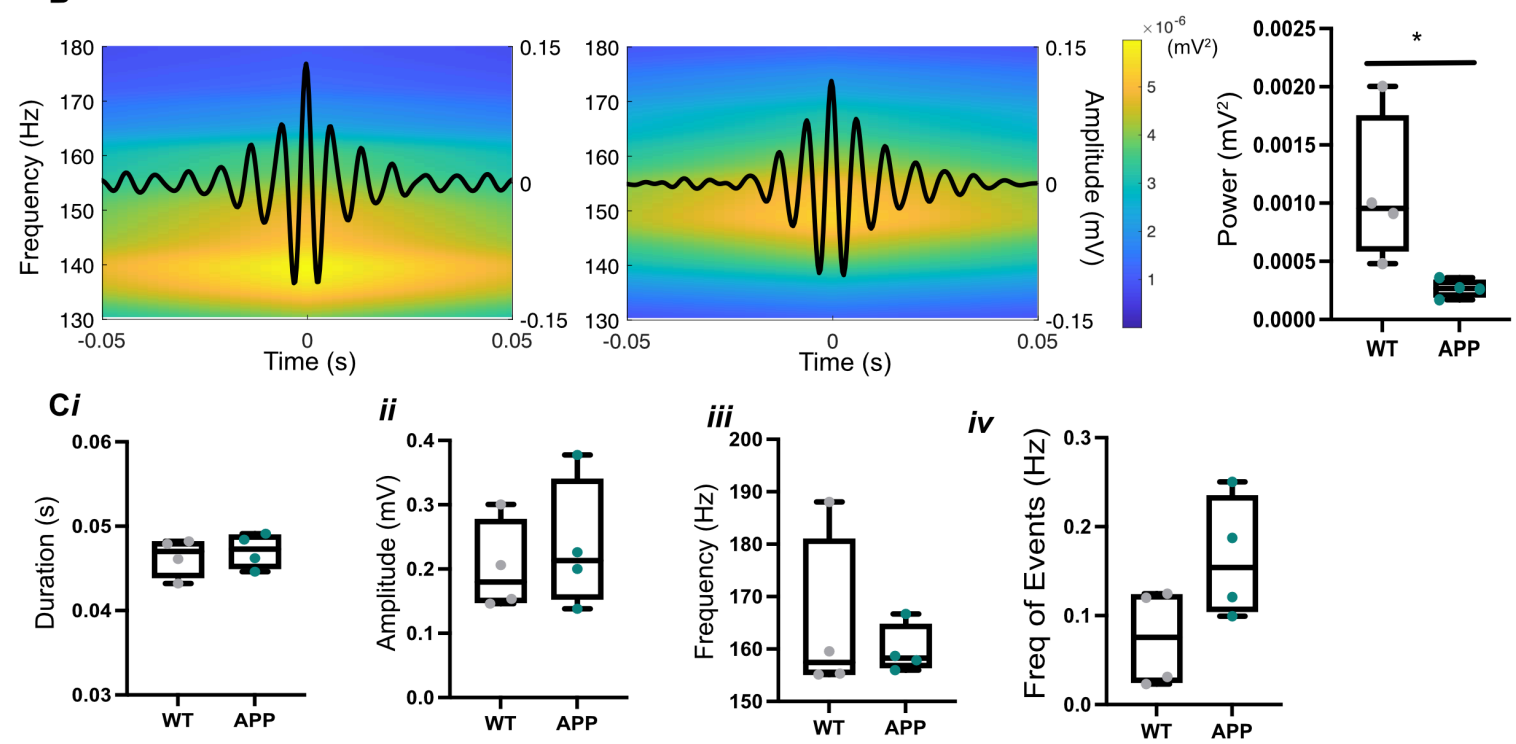

Figure 4 | A decrease in the power of hippocampal ripple events is accompanied by a trending increase in ripple occurrence in APP mice. A Example raw (top) and $130-250 \mathrm{~Hz}$ bandpass filtered (bottom) traces of detected ripple events from WT and APP mice (coloured segments). Scale bars: $20 \mathrm{~ms}, 50 \mu \mathrm{V}$ (bottom), $100 \mu \mathrm{V}$ (top). B Example average ripple traces (black lines) overlaid on corresponding LFP power spectra showing average ripple power. A statistically significant decrease in the average ripple power can be seen in APP mice compared with WT controls (WT: 0.00095 (0.00059-0.00175) vs APP: 0.00027 $(0.00019-0.00034) \mathrm{mV}^{2}, U=0, p=0.02$, Mann-Whitney $U$ test). C No statistically significant difference was found in the average ripple duration (WT: $0.050(0.044-0.048)$ vs APP: $0.047(0.045-0.049) s, U=5, p=0.48$, Mann-Whitney $U$ test) (i), amplitude (WT: 0.18 (0.15 - 0.28) vs APP: $0.21(0.15-0.34) \mathrm{mV}, U=7, p=0.88$, Mann-Whitney $U$ test) (ii) and frequency (WT: 157.4 (155.2 - 180.9) vs APP: 158.3 (156.5 - 164.7) \%, U = 7, p = 0.88, MannWhitney $U$ test) (iii). A trending increase in the occurrence of detected ripple events was found in APP mice compared with WT controls (WT: 0.08 (0.03-0.12) vs APP: 0.15 (0.10- 
0.23) $\mathrm{Hz}, \mathrm{U}=3, \mathrm{p}=0.2$, Mann-Whitney $U$ test) (iv). Box plots show median, IQR and ranges.

Descriptive statistics display median and IQR. $* p<0.05$.

Perisomatic-targeting PV+ interneurons play a key role in generating SWRs ${ }^{72-74}$, and their function is disrupted in several over-expression models of amyloidopathy ${ }^{13-15}$. We thus hypothesised that altered PV+ cell function could account for the decreased ripple power in APP mice. However, immunohistochemical analysis revealed comparable PV+ cell immunoreactivity between APP and WT mice, suggesting that $A \beta$ pathology did not cause PV+ cell loss (Supplementary Figure $\mathbf{4 E}, \boldsymbol{F}$ ). To measure PV+ cell activity, we generated APP and WT mice expressing Cre recombinase in PV+ cells (PVAPP and PVWT mice, respectively) and imaged (Figure 5A) Cre-dependent GCaMP6s in CA1 PV+ cells under consistent, light anaesthesia (Supplementary Figure 5). Most cells (PVAPP = 95.5\%; PVWT = 91.9\%) exhibited spontaneous somatic $\mathrm{Ca}^{2+}$ events indicative of increased neuronal firing ${ }^{51,75}$, that often appeared coordinated between cells in the same field of view (Figure 5B). There was no difference in event frequency or amplitude between PVAPP and PVWT cells (Figure 5C, D). However, $\mathrm{Ca}^{2+}$ activity between simultaneously recorded PV+ cells were more correlated for PVAPP cells compared to PVWT (Figure 5E). This suggests that $A \beta$ pathology increased coordination of firing rate dynamics between PV+ cells without altering overall activity levels. 

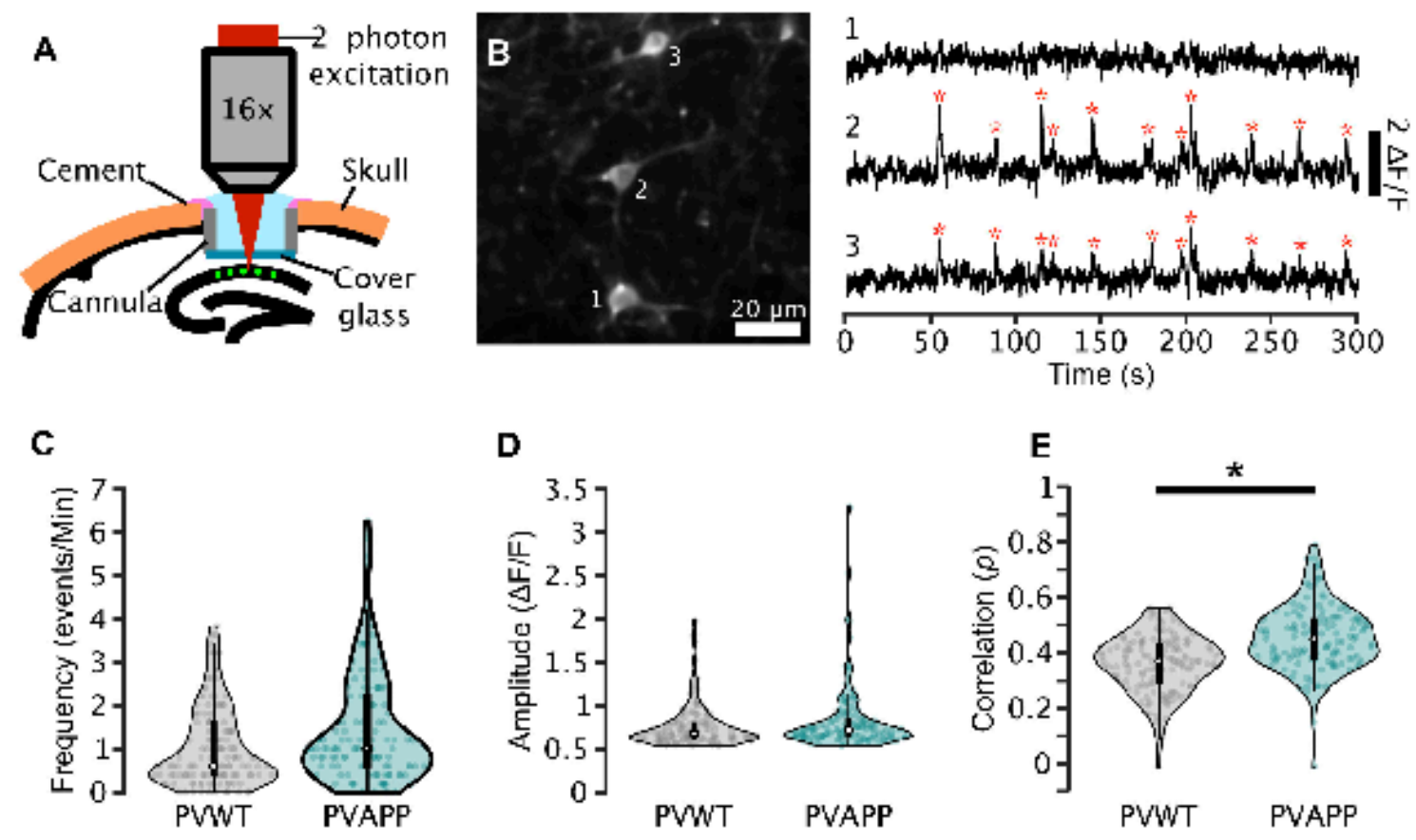

Figure 5 | Consistent levels but increased coordination of PV-expressing interneuron activity in APP mice. A Schematic of two-photon imaging of PV+ neurons (green) in CA1 of the dorsal hippocampus. B Left: Two-photon image of three representative GCaMP6sexpressing cells in a PVWT mouse. Right: GCaMP6s fluorescence traces $(\Delta \mathrm{F} / \mathrm{F})$ recorded from these cells. Red stars denote detected spontaneous Ca ${ }^{2+}$ events. C-D No statistically significant change was found in PVAPP cells ( $N=132$ cells, 28 (13 41) cells/mouse [Med (Min Max)]) compared with PVWT ( $N=135$ cells, 55 (20 60) cells/mouse [Med (Min Max)]) for event frequency (PVWT = 0.61 (1.21) vs PVAPP = 1.01 (1.62) events $/ \mathrm{min}, F(1,245)=0.15$, $\mathrm{p}=0.70)(\mathrm{C})$ or amplitude $(\mathrm{PVWT}=0.66(0.20)$ vs PVAPP $=0.70(0.20) \Delta \mathrm{F} / \mathrm{F}, \mathrm{F}(1,244)=0.12$, $\mathrm{p}=0.73)(\mathrm{D})$. E A statistically significant increase in the zero-lag cross-correlation of $\mathrm{Ca}^{2+}$ activity between simultaneously recorded PV+ cells was found in PVAPP animals compared with PVWT (PVWT $=0.37(0.14)$ vs PVAPP $=0.45(0.14) F(1,260)=4.50, p=0.035)$. Violin plots: marker, median; box, IQR; whiskers, lower-upper adjacent values. Descriptive statistics display median and IQR unless otherwise stated. ${ }^{*} \mathrm{p}<0.05$.

\section{Spindle and ripple coupling to the SWO is unaffected}

Cortical spindles occur at the beginning of the SWO Up states ${ }^{45,76}$. This coupling can be clearly seen when looking at the power of spindles relative to the UDS trace (Figure $6 A$ ) as well as looking at the normalised instantaneous spindle amplitude over UDS phase (Figure $6 \mathbf{B}$ ). To 
assess the coupling strength between UDS and cortical spindles, several different parameters were measured. No significant change was found in the PAC of UDS with cortical spindles when comparing APP with WT (Figure 6B). Additionally, there was no significant change in the time lag between the peak of the SWO Down states and the peak of cortical spindle events (Figure 6C), nor was there a change in the percentage of UDS that were coupled to a cortical spindle (Figure 6D).
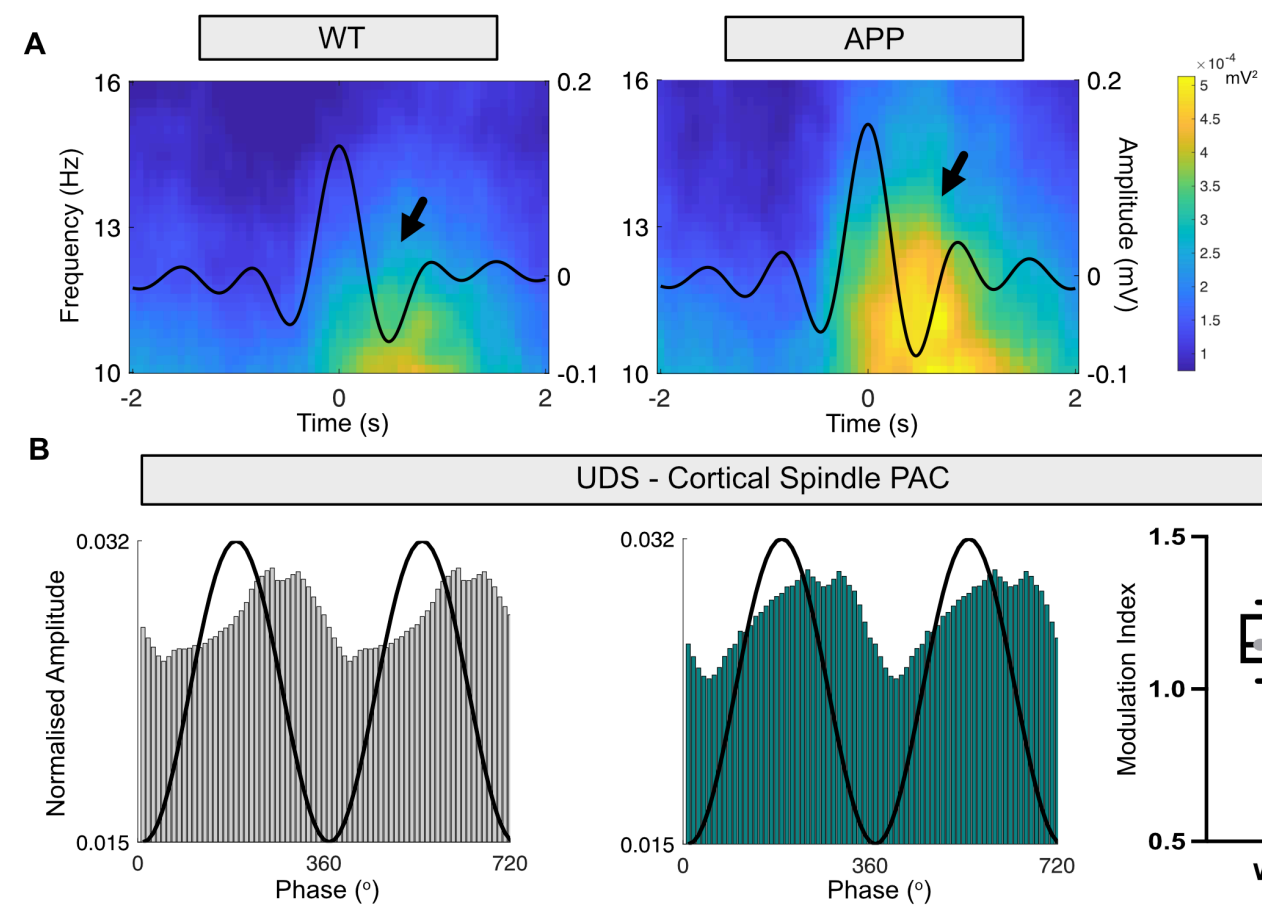

UDS - Cortical Spindle PAC
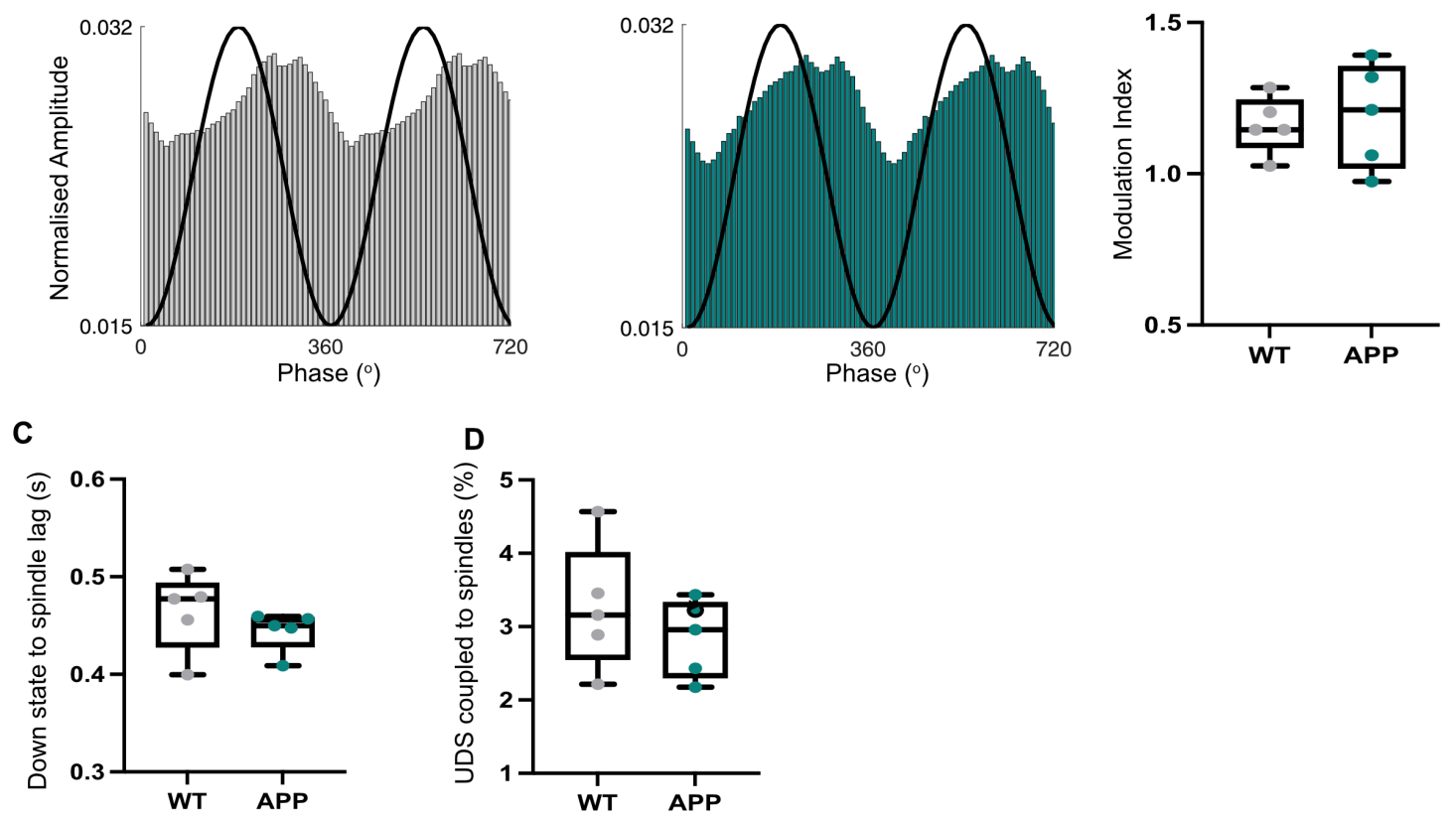

Figure 6 | Coupling of UDS with mPFC spindles is not altered in APP mice. A Example average UDS traces (black lines) overlaid on the corresponding LFP power spectrum showing the power of UDS-locked MPFC spindle oscillations for both WT and APP mice. Spindles can be seen locked to SWO Up states (black arrows). B No statistically significant difference in the PAC of UDS with MPFC spindle oscillations was found in APP animals relative to WT (WT: $1.15(1.09-1.24)$ vs APP: $1.21(1.02-1.36) \mathrm{MI}, \mathrm{U}=10, \mathrm{p}=0.65$, Mann- 
Whitney $U$ test). Shown are example histograms of the change in normalised spindle amplitude (bars) over the phase of the UDS (black line) for both WT (left) and APP (right) mice. C No statistically significant difference was found in the time lag between the peak of the Down state to the peak of the mPFC spindle event (WT: $0.48(0.43-0.49)$ vs APP: $0.45(0.43-0.46) s, U=7, p=0.31$, Mann-Whitney $U$ test). D No statistically significant difference was found in the percentage of UDS coupled to a mPFC spindle event (WT: 3.16 $(2.55-4.01)$ vs APP: $2.96(2.30-3.33) \%, U=9, p=0.54$, Mann-Whitney $U$ test). Box plots show median, IQR and ranges. Descriptive statistics display median and IQR.

Ripples occur towards the end of SWO Up states ${ }^{57}$. We observed an increase in ripple power nested within the trough of concurrent UDS (Figure 7A). No statistically significant change was found in APP animals relative to controls when looking at the PAC of ripples with UDS (Figure $7 \boldsymbol{B}$ ) and when comparing the time lag between the peak of the Down states with the peak of the ripple events (Figure $7 C$ ). However, a trending increase in the percentage of UDS coupled to ripples was found in APP animals compared with WT controls (Figure 7D). This is consistent with the trend towards increased ripple incidence previously reported (see Figure 4Civ). Therefore, although changes occur to local spindle and ripple events, the ability of the SWO to control the temporal precision of neural activity in the MPFC-hippocampal circuit and nest spindle and ripple oscillations within Up states is unaffected in APP animals. 

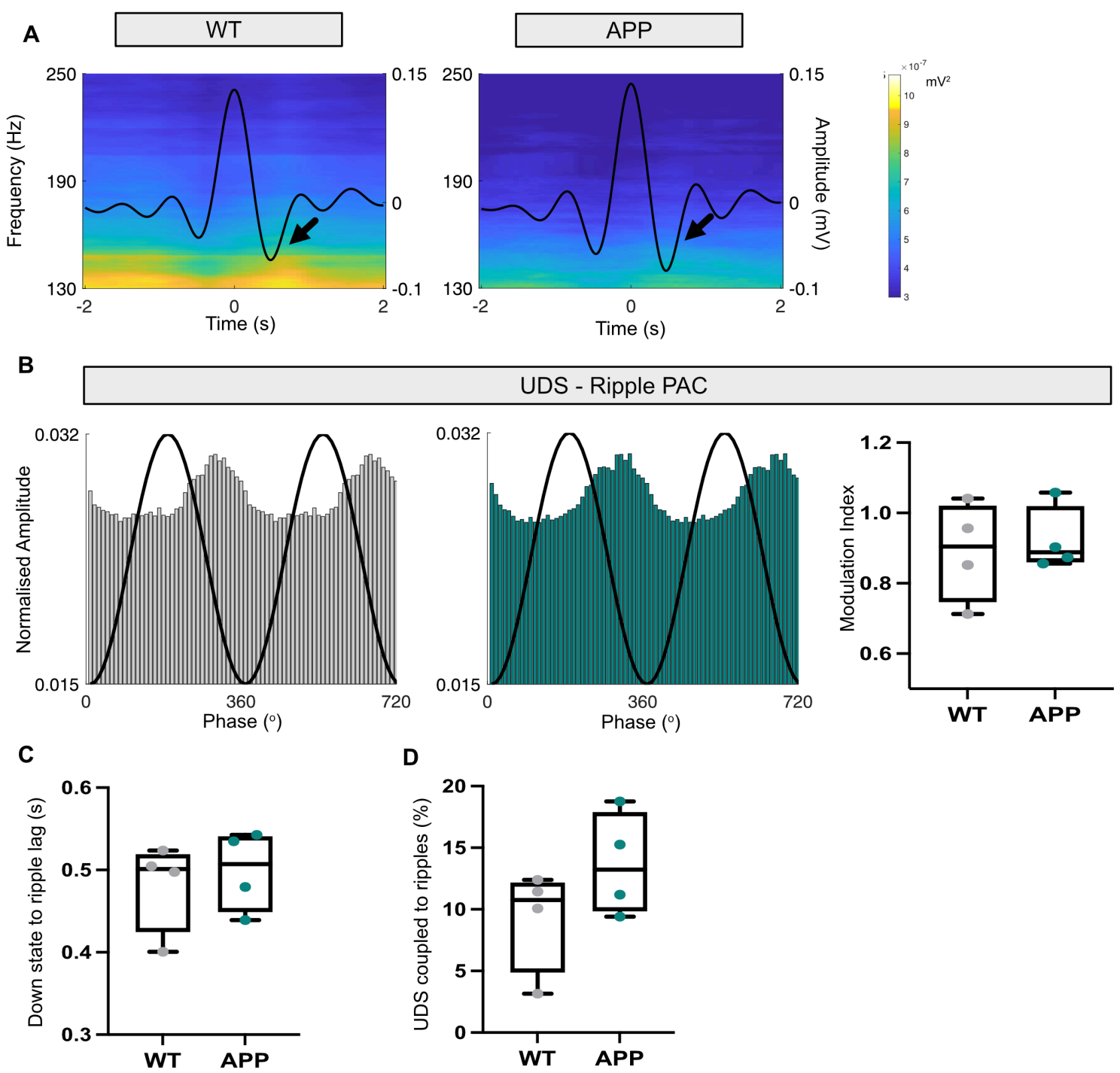

Figure 7 | Coupling of UDS with ripples is not altered in APP mice. A Example average UDS traces (black lines) overlaid on the corresponding LFP power spectrum showing the power of UDS-locked ripple oscillations for both WT and APP mice. Ripples can be seen locked to SWO Up states (black arrows). B No statistically significant difference in the PAC of UDS with ripple oscillations was found between in APP animals compared with WT controls (WT: $0.90(0.75-1.02)$ vs APP: $0.89(0.86-1.02) \mathrm{MI}, U=6, \mathrm{p}=0.68$, Mann-Whitney $U$ test). Shown are example histograms of the changes to the normalised ripple amplitude (bars) over the phase of the UDS (black line) for both WT (left) and APP (right) mice. C No statistically significant difference was found in the time lag between the peak of the Down state to the peak of the ripple event (WT: $0.50(0.42-0.52)$ vs APP: $0.51(0.44-0.54) \mathrm{s}, \mathrm{U}$ $=6, p=0.68$, Mann-Whitney $U$ test). $D$ A trending increase was found in APP mice compared with WT controls when looking at the percentage of UDS coupled to ripples (WT: 10.76 
$(4.89-12.15)$ vs APP: $13.23(9.86-17.88) \%, U=5, p=0.48$, Mann-Whitney $U$ test). Graphs show median and ranges. Box plots show median, IQR and ranges. Descriptive statistics display median and IQR.

Spindle-ripple coupling and the occurrence of the coupling of all three cardinal oscillations together is unaffected

Cortical spindles are initiated before hippocampal ripples, and the temporal coupling of these oscillations helps to drive systems consolidation ${ }^{77-79}$ (Figure $8 \mathbf{A}$ ); it is thought that spindles prime cortical neurons for plasticity-related changes with an increase in intracellular $\mathrm{Ca}^{2+46}$, ready for information to be sent from the hippocampus during a ripple ${ }^{69,70}$. A breakdown in this coupling could therefore have disastrous consequences upon memory. No statistically significant change was found in APP animals relative to WT controls in the time lag between the start of cortical spindles and hippocampal ripples (Figure 8B), nor in the percentage of cortical spindles coupled to ripples (Figure $8 C$ ). This collectively suggests that cortical spindleripple coupling is unaffected in 16-month old APP mice.

Finally, it is the coupling of all 3 cardinal oscillations together that facilitates systems consolidation ${ }^{20,21}$ (Figure 8D). No significant difference was found in the occurrence of UDS with both spindles and ripples when comparing APP animals with WT (Figure 8E). It is interesting to note that despite the changes to MPFC spindles and hippocampal ripples in APP mice, the temporal coordination of the oscillations within the mPFC-hippocampal circuit remains intact. 


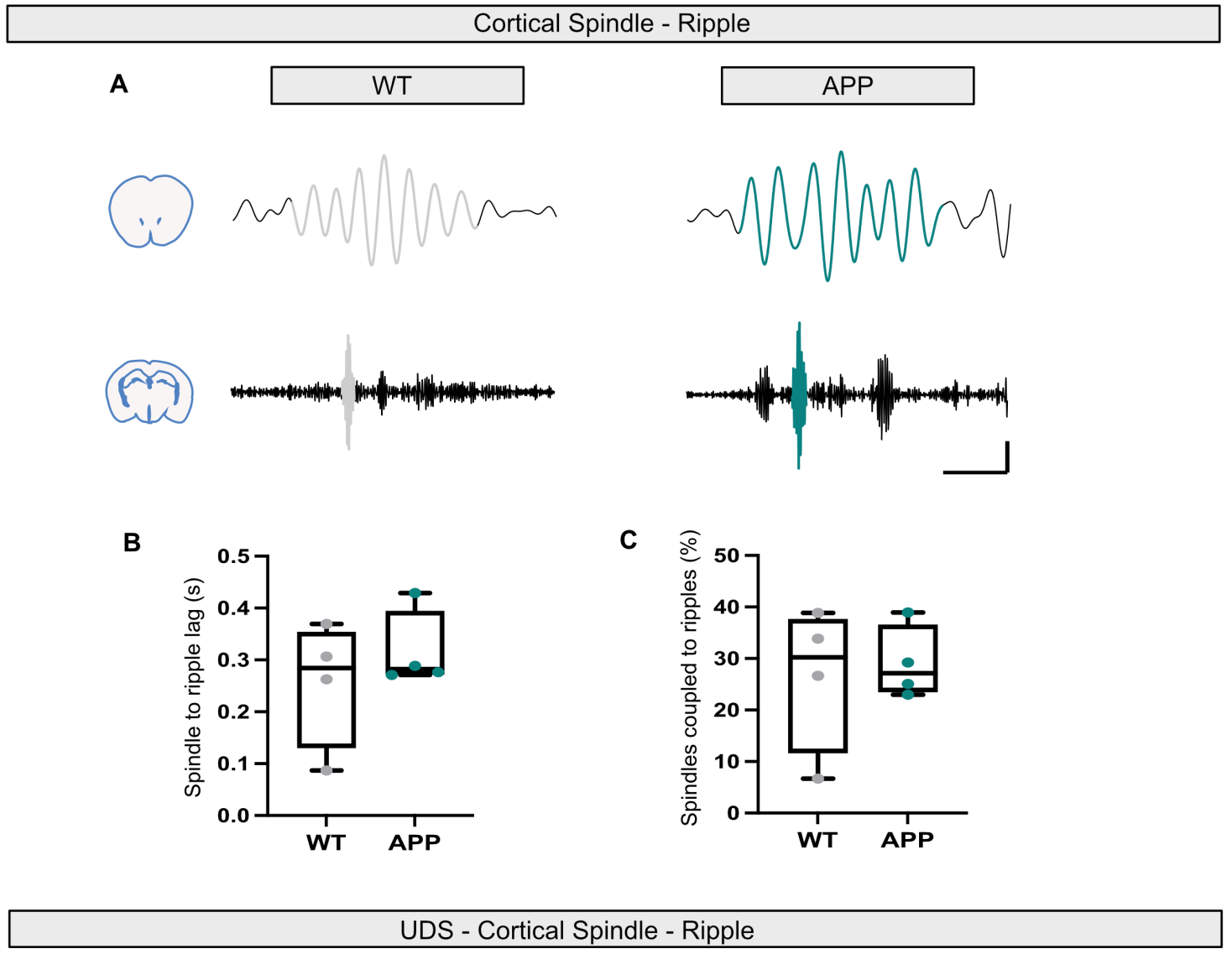

D

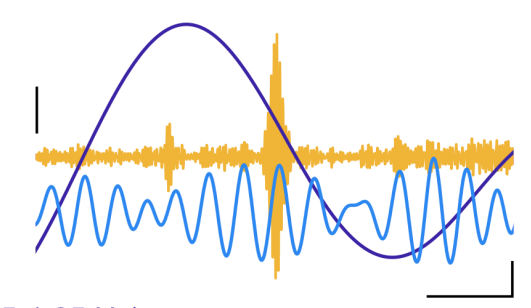

UDS $(0.5-1.25 \mathrm{~Hz})$

Spindle $(11-15 \mathrm{~Hz})$

Ripple $(130-250 \mathrm{~Hz})$

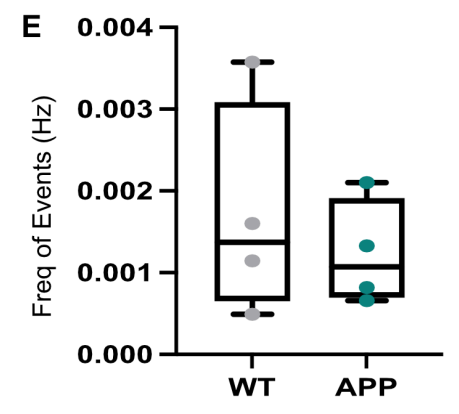

Figure 8 | UDS-spindle-ripple coupling remains intact. A Examples of $11-15 \mathrm{~Hz}$ bandpass filtered traces of spindle events in the MPFC (top) coupled to $130-250 \mathrm{~Hz}$ bandpass filtered CA1 ripple events (bottom) from WT and APP mice (coloured segments). Scale bars: 200 $\mathrm{ms}, 100 \mu \mathrm{V}$. B No statistically significant difference was found in the time lag between the start of mPFC spindle events to the start of CA1 ripple events (WT: $0.28(0.13-0.35)$ vs APP: $0.28(0.27-0.39) \mathrm{s}, U=6, \mathrm{p}=0.68$, Mann-Whitney $U$ test). $C$ No statistically significant difference was found in the percentage of MPFC spindles coupled to CA1 ripples (WT: 30.24 $(11.69-37.61)$ vs APP: $27.16(23.51-36.52) \%, U=8, p=0.99$, Mann-Whitney $U$ test). D Example of bandpass filtered traces showing the three cardinal oscillations occurring together in a WT mouse. UDS $(0.5-1.25 \mathrm{~Hz}$, purple), spindles $(11-15 \mathrm{~Hz}$, blue) and ripples 
(130 - $250 \mathrm{~Hz}$, orange). Scale bars: $200 \mathrm{~ms}, 30 \mu \mathrm{V}$ (right - UDS and spindle), $100 \mu \mathrm{V}$ (left ripple). E No statistically significant difference in the frequency of the three cardinal oscillations occurring together (WT: 0.00066 (0.00065 - 0.00308) vs APP: $0.0011(0.00070$ $-0.00190) \%, U=7, p=0.88$, Mann-Whitney $U$ test). Box plots show median, IQR and ranges. Descriptive statistics display median and IQR. 


\section{Discussion}

This report is the first to comprehensively assess hippocampal-mPFC circuit function during NREM sleep in a mouse model of amyloidopathy, although others have made enquiries along similar lines ${ }^{28,80}$. Changes to cortical spindles as well as hippocampal ripples occurred in the APP $^{\text {NL-G-F }}$ mouse model at sixteen months of age, whereas the SWO appeared to be unimpaired. Additionally, changes to neuronal oscillations measured in local networks were not accompanied by deficits to the temporal coupling between different oscillations throughout the hippocampal-mPFC circuit. Finally, PV+ cells in CA1 were found to exhibit more coordinated activity, and a decrease in PV+ cell density was identified in the ACC. Taken together, these results indicate that $A \beta$ pathology changes $P V+$ cell-mediated inhibition in local neural circuits, causing disruptions to local oscillatory dynamics, but that this does not necessarily disrupt long-range communication between hippocampus and mPFC. These deficits may be a harbinger of subsequent breakdown of hippocampal and mPFC function in AD.

\section{The SWO and nested gamma oscillations are unimpaired}

The SWO and UDS dynamics were unimpaired in sixteen-month-old APP animals, despite widespread amyloid pathology. These results are inconsistent with reported disruptions to the SWO in over-expression mouse models of amyloidopathy ${ }^{24-26}$ and studies that describe a relationship between $A \beta$ load and impaired UDS in humans ${ }^{61}$. Over-expression mouse models tend to develop network deficits at a much younger age $\mathrm{f}^{6,13,31,63}$, so our data suggest that amyloidopathy alone has no effect upon the SWO when hAPP is expressed at physiological levels. The APP ${ }^{\mathrm{NL}-\mathrm{G}-\mathrm{F}}$ mouse is thought to model the preclinical stages of Alzheimer's disease ${ }^{31}$ so at sixteen months old, the phenotype may be too mild to observe changes to the SWO.

Gamma oscillations show reduced power within the cortex and hippocampus of firstgeneration mouse models of amyloidopathy $\mathrm{y}^{13,14,64}$ and there is evidence for impaired CA1 high frequency gamma oscillations during spatial navigation in the APP ${ }^{\text {NL-G-F }}$ model between seven-thirteen months ${ }^{63}$. Gamma oscillations are found nested within SWO Up states ${ }^{59,60}$ and disrupted SWO dynamics in first-generation mouse models can be rescued upon application of $\mathrm{GABA}_{\mathrm{A}}$ receptor agonists ${ }^{24-26,81}$. Therefore, we hypothesised that UDS-nested gamma 
oscillations would be affected during the SWO. However, no statistically significant difference was found in the power of either low or high frequency gamma oscillations or in their coupling to SWO Up states. Although CA1 high frequency gamma oscillations show impairments between seven-thirteen months, a separate study found that MPFC ex vivo gamma oscillations at twelve-months are not $^{82}$. It is therefore possible that like the SWO, A pathology is not sufficiently severe in sixteen month old APP mice to drive impairments to UDS-nested gamma oscillations within the cortex.

\section{An increase in mPFC spindle amplitude and a decrease of PV+ cell density in the ACC}

An increase in the amplitude and power of cortical spindles was identified in APP animals compared to WT controls. Given that PV+ cells within the anterior TRN help generate spindles $^{36}$ and that PV+ cell function is documented to be impaired in first-generation mouse models of $A D^{13,14}$, the density of these interneurons was quantified. No significant difference was found between genotypes. However, this does not necessarily rule out impaired function. Alternatively, there is a statistically significant decrease in the density of PV+ cells in the ACC. PV+ cells in the mPFC help gate incoming excitatory TC input during spindles, and prevent action potential propagation, localising increased $\mathrm{Ca}^{2+}$ to the dendrites to facilitate plasticityrelated changes ${ }^{67}$. A potential loss of these interneurons could impair this function and contribute to the increase in spindle amplitude. If this is the case, the failure of PV+ cells to hold $\mathrm{Ca}^{2+}$ within the dendrites could have disastrous ramifications for plasticity. However, these hypotheses are speculative and need to be investigated.

\section{Disrupted hippocampal ripples and increased $\mathrm{PV}+$ cell coordinated firing}

Ripple events recorded in CA1 Str.P exhibited a statistically significant reduction in power but, interestingly, no change in amplitude. Power is taken from the short-time FFT of the whole event whereas amplitude is the difference between the highest peak and lowest trough, typically located in the centre of the ripple event. This suggests disrupted spike-time dynamics occurring elsewhere in the ripple oscillation. Indeed, CA1 PCs display impaired spiking activity during fast gamma oscillations in the APP ${ }^{\mathrm{NL}-\mathrm{G}-\mathrm{F}}$ mouse model ${ }^{63}$. Both fast gamma oscillations and ripples require the recruitment of PV+ cells to pace PC firing ${ }^{74,83}$; cells that are impaired in first-generation mouse models ${ }^{13,14}$. 
$\mathrm{PV}+$ cells in CA1 were found to have increased coordination of their firing dynamics, yet exhibited no change in their activity levels or density. This increase implies that compensatory alterations are occurring to the CA1 network, potentially as a result of altered neuronal activity $^{6,63,84}$. Moreover, the increased coordination of $P V+$ cell firing could potentially explain the observed decrease in ripple power. PV+ cells are involved in the pacing the ripple oscillation $^{74}$ and follow the spiking of PCs in the ripple trough ${ }^{73}$. An increase in firing coordination can decrease the excitation-inhibition balance during each ripple cycle, resulting in a reduction in power and potential changes to the PV+ cell - PC firing dynamics. This not only has the potential to disturb processes such as spike-time-dependant plasticity, but it could affect the recruitment of neurons for the reactivation of previously encoded information, potentially interfering with consolidation. Future experiments recording the activity of both PCS and PV+ cells during SWRs will give a clearer indication of how these changes occurring to $\mathrm{PV}+$ cells are contributing to dysfunctional oscillatory dynamics. Moreover, given the reduced power of ripples, it would be interesting for future experiments to look at the spiking activity of neurons in the mPFC during a ripple event, to determine if the information contained within the ripple is being effectively transmitted to the cortex ${ }^{69,70}$.

\section{Spared oscillation coupling}

Although impairments can be seen in both cortical spindles and hippocampal ripples, the communication between cardinal oscillations remains intact in sixteen-month-old APP mice. UDS coupling to cortical spindles was unaffected, suggesting that the TC feedback loop that controls their temporal communication is spared ${ }^{44}$. Further indications of spared communication between brain regions comes from the lack of coupling deficits between the SWO and hippocampal ripples, cortical spindles with ripples, as well as the occurrence of all three cardinal oscillations together. The lack of coupling deficits suggest hippocampal-mPFC long-range communication is spared, with the changes occurring to the local oscillations constituting the first features of circuit breakdown.

Finally, learning induces changes to oscillatory activity in the systems consolidation circuit: increased slow wave activity, spindle and ripple density, as well as an increase in spindle- 
ripple coupling are reported ${ }^{40}$. In some studies, $A \beta$-induced deficits to SWR dynamics were only identified during post-learning sleep following a hippocampus-dependant memory task, as opposed to during baseline conditions ${ }^{85,86}$. It is important to assess hippocampal-mPFC circuit function in baseline conditions without prior cognitive loading, however, as learninginduced changes could mask subtle deficits. However, it is possible $A \beta$-induced deficits are more pronounced during active consolidation of a hippocampus-dependant memory. Therefore, repeating experiments under these conditions may produce more striking deficits.

\section{Relevance to hippocampus-dependant memory}

Although changes to local spindle and ripple events were identified, the temporal precision of this circuit was found to be unimpaired. Whether these changes are enough to confer memory impairments is still unknown. Indeed, perturbation of spindle activity can be seen in humans with $A D^{23}$ and disruptions to SWRs can impair hippocampus-dependant memory performance in mice ${ }^{27}$. However, the extent of memory decline in the APP mouse model employed here is a source of debate. The study that first characterised this model identified spatial memory impairments in the Y-maze as early as 6 months of age ${ }^{29,30}$, a finding that others have failed to replicate ${ }^{87}$, potentially due to differences in the environmental enrichment used at different institutions ${ }^{88}$. Additionally, several studies have identified spatial memory impairments from eleven months onwards, yet, these studies all use aversive behavioural paradigms, such as contextual fear conditioning, place avoidance and the Morris water maze $\mathrm{6}^{63,89-91}$. Behaviours that carry negative emotional valence are likely to generate strong memories that only partially reflect the full spectrum of memory function, and therefore arguably do not represent an accurate depiction of memory decline in APPNL-G-F mice ${ }^{92}$. Moreover, the use of inappropriate controls can be found in several studies assessing spatial memory, such as comparisons with other knock-in models (APP ${ }^{\mathrm{NL}-\mathrm{F}}$ and $\mathrm{APP}^{\mathrm{NL}}$ ) and nonlittermate wild-type mice ${ }^{89,90,93,94}$. Behavioural correlates of memory loss are necessary for determining the extent of cognitive impairment. Therefore, a more thorough characterisation of memory decline in APP ${ }^{N L-G-F}$ mice is required, particularly if it is to be considered a valid model of preclinical AD. 


\section{Conclusions}

The fact that impairments are seen to the local oscillations whereas long-range communication is spared potentially points towards the first-steps in the breakdown of hippocampal-mPFC circuit function. Whether these disturbances lead to memory impairments is still to be determined, but it is possible that the changes occurring at this age are a good model of the preclinical phase of AD. Moreover, the changes to PV+ cell density and $\mathrm{Ca}^{2+}$ activity further highlight the effect that pathological amyloid has upon inhibitory neurotransmission, and can be used to explain the alterations occurring to oscillatory activity. Further experimentation is needed to fully decipher the underlying neuronal dysfunction contributing to oscillatory impairments and whether these impairments worsen over time. Nevertheless, this study provides valuable information about how amyloid pathology affects the circuit governing systems consolidation and adds to existing knowledge concerning the pathophysiology of the APP NL-G-F mouse model.

\section{Acknowledgements}

The APPNL-G-F mouse model was kindly supplied by MRC Harwell (UK).

\section{Author Contributions}

The project was conceived and designed by MTC, ESB and JW. All electrophysiological and immunohistochemical experiments and their analysis were conceived and conducted by ESB. Two-photon $\mathrm{Ca}^{2+}$ imaging experiments were conceived by JW and JG and conducted by JG with the help of LA and MTC. TS and TCS provided the APP KI mouse line. Animal lines were bred and maintained by ESB, JG and LA. MTC and JW supervised and assisted the experimental work carried out by ESB. The manuscript was drafted by ESB and all authors discussed the results and critically revised the manuscript for intellectual content.

\section{Funding}

This work was supported by an Alzheimer's Research UK Interdisciplinary research grant ARUK-IRG2017B-4 (Michael T Craig), an Alzheimer's Research UK Fellowship ARUK-RF2015-6 (Jonathan Witton), an Alzheimer's Society Senior Fellowship AS-JF-14-007 (Francesco Tamagnini), and a Northcott Devon Medical Foundation grant TB/MG/NO5002 (Craig / Brady / Andrianova). Erica Brady was a GW4 BioMed doctoral training program student funded by 
the Medical Research Council (MR/N0137941/1), awarded to the Universities of Bath, Bristol, Cardiff and Exeter. Salary support for Lilya Andrianova was provided by Biotechnology and Biological Sciences Research Council grant BB/P001475/1 (Michael T Craig). The 2 photon imaging facilities were supported by Wellcome Trust multi-user equipment grant 202936/Z/16/Z (Andrew D Randall).

\section{Competing interests}

All authors report no competing interests.

\section{References}

1 Nichols E, Szoeke CEI, Vollset SE, Abbasi N, Abd-Allah F, Abdela J et al. Global, regional, and national burden of Alzheimer's disease and other dementias, 1990-2016: a systematic analysis for the Global Burden of Disease Study 2016. The Lancet Neurology 2019; 18: 88.

2 Busche MA, Chen X, Henning HA, Reichwald J, Staufenbiel M, Sakmann B et al. Critical role of soluble amyloid- $\beta$ for early hippocampal hyperactivity in a mouse model of Alzheimer's disease. PNAS 2012; 109: 8740-8745.

3 Busche MA, Eichhoff G, Adelsberger H, Abramowski D, Wiederhold K-H, Haass C et al. Clusters of Hyperactive neurons Near Amyloid Plaques in a mouse model of Alzheimer's disease. Science 2008; 321: 1686-1689.

4

Hsieh H, Boehm J, Sato C, Iwatsubo T, Tomita T, Sisodia S et al. AMPAR Removal Underlies A $\beta$-Induced Synaptic Depression and Dendritic Spine Loss. Neuron 2006; 52: 831-843.

5 Shankar GM, Bloodgood BL, Townsend M, Walsh DM, Selkoe DJ, Sabatini BL. Natural oligomers of the Alzheimer amyloid- $\beta$ protein induce reversible synapse loss by modulating an NMDA-type glutamate receptor-dependent signaling pathway. Journal of Neuroscience 2007; 27: 2866-2875.

6 Palop J, Chin J, Roberson ED, Wang J, Thwin MT, Bien-ly N et al. Aberrant Excitatory Neuronal Activity and Compensatory Remodeling of Inhibitory Hippocampal Circuits in Mouse Models of Alzheimer's Disease. Neuron 2007; 55: 697-711. 
7 Chung $\mathrm{H}$, Park $\mathrm{K}$, Jang $\mathrm{HJ}$, Kohl MM, Kwag J. Dissociation of somatostatin and parvalbumin interneurons circuit dysfunctions underlying hippocampal theta and gamma oscillations impaired by amyloid $\beta$ oligomers in vivo. Brain Structure and Function 2020; 225: 935-954.

8 Sperling RA, LaViolette PS, O’Keefe K, O’Brien J, Rentz DM, Pihlajamaki M et al. Amyloid Deposition Is Associated with Impaired Default Network Function in Older Persons without Dementia. Neuron 2009; 63: 178-188.

9 Bakker A, Krauss GL, Albert MS, Speck CL, Jones LR, Stark CE et al. Reduction of Hippocampal Hyperactivity Improves Cognition in Amnestic Mild Cognitive Impairment. Neuron 2012; 74: 467-474.

10 Born HA. Seizures in Alzheimer's disease. Neuroscience. 2015; 286: 251-263.

11 Dauwels J, Srinivasan K, Reddy MR, Musha T, Vialatte F-B, Latchoumane C et al. Slowing and Loss of Complexity in Alzheimer's EEG: Two Sides of the Same Coin? International Journal of Alzheimer's Disease 2011; 2011: 1-10.

12 Palop J, Mucke L. Network abnormalities and interneuron dysfunction in Alzheimer disease. Nature reviews Neuroscience 2016; 17: 777-792.

13 Verret L, Mann EO, Hang GB, Barth AMI, Cobos I, Ho K et al. Inhibitory Interneuron Deficit Links Altered Network Activity and Cognitive Dysfunction in Alzheimer Model. CELL 2012; 149: 708-721.

14 Martinez-Losa M, Tracy TE, Ma K, Verret L, Clemente-Perez A, Khan AS et al. Nav1.1Overexpressing Interneuron Transplants Restore Brain Rhythms and Cognition in a Mouse Model of Alzheimer's Disease. Neuron 2018; 98: 1-15.

15 Hijazi S, Heistek TS, Scheltens P, Neumann U, Shimshek DR, Mansvelder HD et al. Early restoration of parvalbumin interneuron activity prevents memory loss and network hyperexcitability in a mouse model of Alzheimer's disease. Molecular Psychiatry 2019; 25: 3380-3398.

16 Bubu O, Brannick M, Mortimer J, Umasabor-Bubu O, Sebastião Y, Wen Y et al. Sleep, Cognitive impairment, and Alzheimer's disease: A Systematic Review and MetaAnalysis. Sleep. 2017; 40: 1-18.

17 Sindi S, Kåreholt I, Johansson L, Skoog J, Sjöberg L, Wang HX et al. Sleep disturbances and dementia risk: A multicenter study. Alzheimer's and Dementia 2018; 14: 12351242. 
18 Sigurdsson T, Duvarci S. Hippocampal-Prefrontal Interactions in Cognition, Behavior and Psychiatric Disease. Frontiers in Systems Neuroscience 2015; 9: 190.

19 Rasch B, Born J. About sleep's role in memory. Physiological Reviews 2013; 93: 681766.

20 Crunelli V, Hughes SW. The slow $(<1 \mathrm{~Hz})$ rhythm of non-REM sleep: a dialogue between three cardinal oscillators. Nature Neuroscience 2010; 13: 9-17.

21 Maingret N, Girardeau G, Todorova R, Goutierre M, Zugaro M. Hippocampo-cortical coupling mediates memory consolidation during sleep. Nature Neuroscience 2016; 19 : 959-964.

22 Mander BA, Marks SM, Vogel JW, Rao V, Lu B, Saletin JM et al. $\beta$-amyloid disrupts human NREM slow waves and related hippocampus-dependent memory consolidation. Nature Neuroscience 2015; 18: 1051-1057.

23 Kam K, Parekh A, Sharma RA, Andrade A, Lewin M, Castillo B et al. Sleep oscillationspecific associations with Alzheimer's disease CSF biomarkers: Novel roles for sleep spindles and tau. Molecular Neurodegeneration 2019; 14: 1-12.

24 Castano-Prat P, Perez-Mendez L, Perez-Zabalza M, Sanfeliu C, Giménez-Llort L, Sanchez-Vives M v. Altered slow (\&lt;1 Hz) and fast (beta and gamma) neocortical oscillations in the 3xTg-AD mouse model of Alzheimer's disease under anesthesia. Neurobiology of Aging 2019; 79: 142-151.

25 Busche MA, Kekuš M, Adelsberger H, Noda T, Förstl H, Nelken I et al. Rescue of longrange circuit dysfunction in Alzheimer's disease models. Nature neuroscience 2015; 18: 1623-30.

26 Kastanenka K v, Hou SS, Shakerdge N, Logan R, Feng D, Wegmann S et al. Optogenetic Restoration of Disrupted Slow Oscillations Halts Amyloid Deposition and Restores Calcium Homeostasis in an Animal Model of Alzheimer's Disease. PLOS ONE 2017; 12: $1-25$.

27 Caccavano A, Bozzelli PL, Forcelli PA, Pak DTS, Wu J-Y, Conant K et al. Inhibitory Parvalbumin Basket Cell Activity is Selectively Reduced during Hippocampal Sharp Wave Ripples in a Mouse Model of Familial Alzheimer's Disease. Journal of Neuroscience 2020; 40: 5116-5136. 
28 Zhurakovskaya E, Ishchenko I, Gureviciene I, Aliev R, Gröhn O, Tanila H. Impaired hippocampal-cortical coupling but preserved local synchrony during sleep in APP/PS1 mice modeling Alzheimer's disease. Scientific Reports 2019; 9: 5380.

29 Nilsson P, Saito T, Saido TC. New Mouse Model of Alzheimer's. ACS Chemical Neuroscience 2014; 5: 499-502.

30 Saito T, Matsuba Y, Mihira N, Takano J, Nilsson P, Itohara S et al. Single App knock-in mouse models of Alzheimer's disease. Nature Neuroscience 2014; 17: 661-664.

31 Sasaguri H, Nilsson P, Hashimoto S, Nagata K, Saito T, de Strooper B et al. APP mouse models for Alzheimer's disease preclinical studies. The EMBO journal 2017; 36: 24732487.

32 van de Werd HJJM, Rajkowska G, Evers P, Uylings HBM. Cytoarchitectonic and chemoarchitectonic characterization of the prefrontal cortical areas in the mouse. Brain Structure and Function 2010; 214: 339-353.

33 Buzsaki G, Horvath Z, Urioste R, Hetke J, Wise K. High-Frequency Network Oscillation in the Hippocampus. Science 1992; 256: 1025-1027.

34 Dombeck DA, Harvey CD, Tian L, Looger LL, Tank DW. Functional imaging of hippocampal place cells at cellular resolution during virtual navigation. Nature Neuroscience 2010; 13: 1433-1440.

35 Pologruto TA, Sabatini BL, Svoboda K. Scanlmage: Flexible software for operating laser scanning microscopes. BioMedical Engineering Online 2003; 2: 1-9.

36 Latchoumane CF v., Ngo HV v., Born J, Shin HS. Thalamic Spindles Promote Memory Formation during Sleep through Triple Phase-Locking of Cortical, Thalamic, and Hippocampal Rhythms. Neuron 2017; 95: 424-435.e6.

37 Adamantidis A, Lüthi A. Optogenetic Dissection of Sleep-Wake States In Vitro and In Vivo. In: Handbook of Experimental Pharmacology. Springer New York LLC, 2019, pp 125-151.

38 Kohto S, Taguchil Y, Matsumoto N, Wada M, Huang Z-L, Urade Y. Algorithm for sleep scoring in experimental animals based on fast Fourier transform power spectrum analysis of the electroencephalogram. Sleep and Biological Rhythms 2008; 6: 163-171.

39 Witton J, Staniaszek LE, Bartsch U, Randall AD, Jones MW, Brown JT. Disrupted hippocampal sharp-wave ripple-associated spike dynamics in a transgenic mouse model of dementia. The Journal of physiology 2016; 594: 4615-30. 
40 Mölle M, Eschenko O, Gais S, Sara SJ, Born J. The influence of learning on sleep slow oscillations and associated spindles and ripples in humans and rats. European Journal of Neuroscience 2009; 29: 1071-1081.

41 Chauvette S, Crochet S, Volgushev M, Timofeev I. Properties of slow oscillation during slow-wave sleep and anesthesia in cats. Journal of Neuroscience 2011; 31: 1499815008.

42 Nir Y, Staba RJ, Andrillon T, Vyazovskiy V v., Cirelli C, Fried l et al. Regional Slow Waves and Spindles in Human Sleep. Neuron 2011; 70: 153-169.

43 Phillips KG, Bartsch U, McCarthy AP, Edgar DM, Tricklebank MD, Wafford KA et al. Decoupling of Sleep-Dependent Cortical and Hippocampal Interactions in a Neurodevelopmental Model of Schizophrenia. Neuron 2012; 76: 526.

44 David F, Schmiedt JT, Taylor HL, Orban G, di Giovanni G, Uebele VN et al. Essential thalamic contribution to slow waves of natural sleep. The Journal of Neuroscience 2013; 33: 19599-610.

45 Staresina BP, Bergmann TO, Bonnefond M, van der Meij R, Jensen O, Deuker L et al. Hierarchical nesting of slow oscillations, spindles and ripples in the human hippocampus during sleep. Nature Neuroscience 2015; 18: 1679-1686.

46 Niethard N, Ngo H-V v, Ehrlich I, Born J, Hertie D. Cortical circuit activity underlying sleep slow oscillations and spindles. PNAS 2018; 115: 9220-9229.

47 Bartsch U, Simpkin AJ, Demanuele C, Wamsley E, Marston HM, Jones MW. Distributed slow-wave dynamics during sleep predict memory consolidation and its impairment in schizophrenia. npj Schizophrenia 2019; 5: 1-11.

48 Canolty RT, Edwards E, Dalal SS, Soltani M, Nagarajan SS, Kirsch HE et al. High Gamma Power Is Phase-Locked to Theta Oscillations in Human Neocortex. Science 2006; 313: 1626-1628.

49 Schindelin J, Arganda-Carreras I, Frise E, Kaynig V, Longair M, Pietzsch T et al. Fiji: an open-source platform for biological-image analysis. Nature Methods 2012; 9: 676-682.

50 Fienup JR, Guizar-Sicairos M, Thurman ST. Efficient subpixel image registration algorithms. Optics Letters 2008; 33: 156-158.

51 Chen T-W, Wardill TJ, Sun Y, Pulver SR, Renninger SL, Baohan A et al. Ultrasensitive fluorescent proteins for imaging neuronal activity. Nature 2013; 499: 295-300. 
52 Aarts E, Verhage M, Veenvliet J V, Dolan C V, van der Sluis S. A solution to dependency: using multilevel analysis to accommodate nested data. Nature neuroscience 2014; 17 : 491-496.

53 Amzica F, Steriade M. Electrophysiological correlates of sleep delta waves. Electroencephalography and Clinical Neurophysiology 1998; 107: 69-83.

54 Sirota A, Csicsvari J, Buhl D, Buzsáki G. Communication between neocortex and hippocampus during sleep in rodents. PNAS 2003; 100: 2065-9.

55 Varela C, Wilson MA. Mpfc spindle cycles organize sparse thalamic activation and recently active ca1 cells during non-rem sleep. eLife 2020; 9: 1-34.

56 Kim J, Gulati T, Ganguly K. Competing Roles of Slow Oscillations and Delta Waves in Memory Consolidation versus Forgetting. Cell 2019; 179: 514-526.e13.

57 Mölle M, Yeshenko O, Marshall L, Sara SJ, Born J. Hippocampal sharp wave-ripples linked to slow oscillations in rat slow-wave sleep. Journal of Neurophysiology 2006; 96 : $62-70$.

58 Steriade M, Nunez A, Amzica F. A Novel Slow $(<1 \mathrm{~Hz})$ Oscillation of Neocortical neurons in vivo: Depolarizing and Hyperpolariziong Components. The Journal of Neuroscience 1993; 73: 3252-3285.

59 Steriade M, Contreras D, Amzica F, Timofeev L. Synchronization of Fast $(30-40 \mathrm{~Hz})$ Spontaneous Oscillations in Intrathalamic and Thalamocortical Networks. The Journal of Neuroscience 1996; 76: 2788-2808.

60 Valderrama M, Crépon B, Botella-Soler V, Martinerie J, Hasboun D, Alvarado-Rojas C et al. Human Gamma Oscillations during Slow Wave Sleep. PLoS ONE 2012; 7: e33477.

61 Mander BA, Marks SM, Vogel JW, Rao V, Lu B, Saletin JM et al. $\beta$-amyloid disrupts human NREM slow waves and related hippocampus-dependent memory consolidation. Nature Neuroscience 2015; 18: 1051-1057.

62 Murty DVPS, Manikandan K, Kumar WS, Ramesh RG, Purokayastha S, Nagendra B et al. Stimulus-induced gamma rhythms are weaker in human elderly with mild cognitive impairment and Alzheimer's disease. elife 2021; 10.

63 Jun H, Bramian A, Soma S, Saito T, Saido TC, Igarashi KM. Disrupted Place Cell Remapping and Impaired Grid Cells in a Knockin Model of Alzheimer's Disease. Neuron 2020; 107: 1-18. 
64 Driver JE, Racca C, Cunningham MO, Towers SK, Davies $\mathrm{CH}$, Whittington MA et al. Impairment of hippocampal gamma $(\gamma)$-frequency oscillations in vitro in mice overexpressing human amyloid precursor protein (APP). European Journal of Neuroscience 2007; 26: 1280-1288.

65 Lüthi A. What Are Sleep Spindle Waves? Sleep Spindles: Where They Come From, What They Do. The Neuroscientist 2014; 20: 243-256.

66 Clemente-Perez A, Makinson SR, Higashikubo B, Brovarney S, Cho FS, Urry A et al. Distinct Thalamic Reticular Cell Types Differentially Modulate Normal and Pathological Cortical Rhythms. Cell Reports 2017; 19: 2130-2142.

67 Delevich K, Tucciarone J, Huang ZJ, Li B. The mediodorsal thalamus drives feedforward inhibition in the anterior cingulate cortex via parvalbumin interneurons. Journal of Neuroscience 2015; 35: 5743-53.

68 Nádasdy Z, Hirase H, Czurkó A, Csicsvari J, Buzsáki G. Replay and time compression of recurring spike sequences in the hippocampus. Journal of Neuroscience 1999; 19: 9497-9507.

69 Wierzynski CM, Lubenov E v., Gu M, Siapas AG. State-Dependent Spike-Timing Relationships between Hippocampal and Prefrontal Circuits during Sleep. Neuron 2009; 61: 587-596.

70 Dong X, Wang V, Ikemoto XS. Coordinated Interaction between Hippocampal SharpWave Ripples and Anterior Cingulate Unit Activity. Journal of Neuroscience 2016; 36: 10663-10672.

71 Girardeau G, Zugaro M. Hippocampal ripples and memory consolidation. Current Opinion in Neurobiology. 2011; 21: 452-459.

72 Ognjanovski N, Schaeffer S, Wu J, Mofakham S, Maruyama D, Zochowski M et al. Parvalbumin-expressing interneurons coordinate hippocampal network dynamics required for memory consolidation. Nature Communications 2017; 8.

73 Stark E, Roux L, Eichler R, Senzai Y, Royer S, Buzsáki G. Pyramidal cell-interneuron interactions underlie hippocampal ripple oscillations. Neuron 2014; 83: 467-480.

74 Gan J, Weng S ming, Pernía-Andrade AJ, Csicsvari J, Jonas P. Phase-Locked Inhibition, but Not Excitation, Underlies Hippocampal Ripple Oscillations in Awake Mice In Vivo. Neuron 2017; 93: 308-314. 
75 Ali F, Kwan AC. Interpreting in vivo calcium signals from neuronal cell bodies, axons, and dendrites: a review. Neurophotonics 2020; 7: 1.

76 Mölle M, Marshall L, Gais S, Born J. Grouping of spindle activity during slow oscillations in human non-rapid eye movement sleep. Journal of Neuroscience 2002; 22: 1094110947.

77 Siapas AG, Wilson MA. Coordinated Interactions between Hippocampal Ripples and Cortical Spindles during Slow-Wave Sleep. Neuron 1998; 21: 1123-1128.

78 Clemens Z, Molle M, Eross L, Barsi P, Halasz P, Born J. Temporal coupling of parahippocampal ripples, sleep spindles and slow oscillations in humans. Brain 2007; 130: $2868-2878$.

79 Xia F, Richards BA, Tran MM, Josselyn SA, Takehara-Nishiuchi K, Frankland PW. Parvalbumin-positive interneurons mediate neocortical-hippocampal interactions that are necessary for memory consolidation. eLife 2017; 6. doi:10.7554/eLife.27868.

80 Benthem SD, Skelin I, Moseley SC, Stimmell AC, Dixon JR, Melilli AS et al. Impaired Hippocampal-Cortical Interactions during Sleep in a Mouse Model of Alzheimer's Disease. Current Biology 2020; 30: 2588-2601.e5.

81 Buzsaki G, Wang X-J. Mechanisms of Gamma Oscillations. Annual review of neuroscience 2012; 6: 2166-2171.

82 Pervolaraki E, Hall SP, Foresteire D, Saito T. Insoluble A $\beta$ overexpression in an App knock-in mouse model alters microstructure and gamma oscillations in the prefrontal cortex, affecting anxiety-related behaviours. Disease Models and Mechanisms 2019; 12.

83 Sohal VS, Zhang F, Yizhar O, Deisseroth K. Parvalbumin neurons and gamma rhythms enhance cortical circuit performance. Nature 2009; 459: 698-702.

84 Hollnagel J-O, Elzoheiry S, Gorgas K, Kins S, Beretta CA, Kirsch J et al. Early alterations in hippocampal perisomatic GABAergic synapses and network oscillations in a mouse model of Alzheimer's disease amyloidosis. PLOS ONE 2019; 14: e0209228.

85 Nicole O, Hadzibegovic S, Gajda J, Bontempi B, Bem T, Meyrand P. Soluble amyloid beta oligomers block the learning-induced increase in hippocampal sharp wave-ripple rate and impair spatial memory formation. Scientific Reports 2016; 6: 22728.

86 Jura B, Macrez N, Meyrand P, Bem T. Deficit in hippocampal ripples does not preclude spatial memory formation in APP/PS1 mice. Scientific Reports 2019; 9: 1-12. 
87 Whyte LS, Hemsley KM, Lau AA, Hassiotis S, Saito T, Saido TC et al. Reduction in open field activity in the absence of memory deficits in the App NL-G-F knock-in mouse model of Alzheimer's disease. Behavioural Brain Research 2018; 336: 177-181.

88 Bayne K. Environmental enrichment and mouse models: Current perspectives. Animal Models and Experimental Medicine 2018; 1: 82.

89 Masuda A, Kobayashi Y, Kogo N, Saito T, Saido TC, Itohara S. Cognitive deficits in single App knock-in mouse models. Neurobiology of learning and memory 2016; 135: 73-82.

90 Latif-Hernandez A, Shah D, Craessaerts K, Saido T, Saito T, de Strooper B et al. Subtle behavioral changes and increased prefrontal-hippocampal network synchronicity in APPNL-G-F mice before prominent plaque deposition. Behavioural Brain Research 2019; 364: 431-441.

91 Maezono SEB, Kanuka M, Tatsuzawa C, Morita M, Kawano T, Kashiwagi M et al. Progressive changes in sleep and its relations to amyloid- $\beta$ distribution and learning in single app knock-in mice. eNeuro 2020; 7./pmc/articles/PMC7196722/ (accessed 17 Mar2021).

92 McGaugh JL. Making lasting memories: Remembering the significant. PNAS 2013; 110: 10402.

93 Sakakibara Y, Sekiya M, Saito T, Saido TC, lijima KM. Cognitive and emotional alterations in App knock-in mouse models of $A \beta$ amyloidosis. BMC neuroscience 2018; 19: 46 .

94 Jacob S, Davies G, de Bock M, Hermans B, Wintmolders C, Bottelbergs A et al. Neural oscillations during cognitive processes in an App knock-in mouse model of Alzheimer's disease pathology. Scientific reports 2019; 9: 16363. 